\title{
Elongator controls cortical interneuron migration by regulating actomyosin dynamics
}

\author{
Sylvia Tielens ${ }^{1,2, *}$, Sandra Huysseune ${ }^{1,2, *}$, Juliette D Godin ${ }^{1,2}$, Alain Chariot ${ }^{2,3,4}$, Brigitte Malgrange ${ }^{1,2}$, \\ Laurent Nguyen ${ }^{1,2}$ \\ ${ }^{I}$ GIGA-Neurosciences, 4000 Liège, Belgium, ${ }^{2}$ Interdisciplinary Cluster for Applied Genoproteomics (GIGA-R), 4000 Liège, Bel- \\ gium, ${ }^{3}$ GIGA-Molecular Biology of Diseases, 4000 Liège, Belgium, ${ }^{4}$ Walloon Excellence in Lifesciences and Biotechnology (WEL- \\ BIO), University of Liège, CHU Sart Tilman, 4000 Liège, Belgium
}

The migration of cortical interneurons is a fundamental process for the establishment of cortical connectivity and its impairment underlies several neurological disorders. During development, these neurons are born in the ganglionic eminences and they migrate tangentially to populate the cortical layers. This process relies on various morphological changes that are driven by dynamic cytoskeleton remodelings. By coupling time lapse imaging with molecular analyses, we show that the Elongator complex controls cortical interneuron migration in mouse embryos by regulating nucleokinesis and branching dynamics. At the molecular level, Elongator fine-tunes actomyosin forces by regulating the distribution and turnover of actin microfilaments during cell migration. Thus, we demonstrate that Elongator cell-autonomously promotes cortical interneuron migration by controlling actin cytoskeletal dynamics.

Keywords: cortical interneurons; cerebral cortex; actomyosin; cofilin

Cell Research (2016) 26:1131-1148. doi:10.1038/cr.2016.112; published online 27 September 2016

\section{Introduction}

The cerebral cortex is an evolutionarily advanced structure of the brain that computes higher cognitive functions [1]. Its laminar organization arises inside-out as cortical progenitors generate successive waves of excitatory projection neurons in the pallium [2] and inhibitory interneurons in the subpallium [3]. The cortical interneurons are heterogeneous and originate from three main sources in the subpallium: the medial and caudal ganglionic eminences (MGEs and CGEs, respectively) and the preoptic area (POA) $[4,5]$. They navigate along tangential routes to reach the cortical plate (reviewed in [6]) where they adopt a multidirectional mode of migration to settle at their final laminar position [7]). Interneurons undergo a saltatory migration that involves cycles of cellular events, starting with the extension and branching of their leading process. This process steers the inter-

*These two authors contributed equally to this work.

Correspondence: Laurent Nguyen

E-mail: lnguyen@ulg.ac.be

Received 24 December 2015; revised 27 June 2016; accepted 11 August 2016; published online 27 September 2016 neuron within the forebrain parenchyma by sensing the surrounding microenvironment $[8,9]$. Interneurons subsequently undergo nucleokinesis and retract their trailing process, which ultimately leads to the net movement of the cell $[10,11]$. These modifications in cell shape are driven by the dynamic remodeling of the cytoskeleton [12], which is composed of actin microfilaments, microtubules (MTs) and intermediate filaments. Mutations that affect cytoskeleton regulation or integrity often lead to cortical malformations associated with neurological disorders $[13,14]$.

Our recent investigation of the Elongator complex (Elp1-Elp6) revealed its expression within the developing brain [15], and its mutations have been associated with neurological disorders, such as familial dysautonomia and amyotrophic lateral sclerosis [16-19]. The complex comprises important subunits, including the Elp1 scaffold subunit and the Elp3 enzymatic core that contains a lysine acetyltransferase domain (KAT) [20-22] and a demethylation domain (SAM) [23]. Elongator possesses distinct functions in the nucleus and the cytoplasm (reviewed in [17]). Its nuclear roles include transcription elongation $[24,25]$ and paternal genome demethylation [23]. In contrast, its main cytoplasmic role, described in 
yeast [26-28] and in mammals [29], is the promotion of tRNA modifications at the first nucleotide anticodon U34 (Elongator belongs to tRAMEs, an enzymatic cascade that promotes U34 tRNA anticodon side chain modifications) [30], thereby controlling mRNA translational efficiency in health [27] and disease [31]. Loss of Elongator activity leads to slowdown of translation resulting in proteotoxic stress [27]. In the nervous system, Elongator controls the fate of cortical progenitors by ensuring proper tRNA modifications [29], promotes the migration and maturation of projection neurons by facilitating the acetylation of tubulin [15], and fine-tunes the neurotransmission at the neuromuscular junction in fly [32]. In addition, Elongator supports target tissue innervation and survival of sensory and sympathetic neurons [33, 34].

The present work demonstrates the role of Elongator in interneuron migration during corticogenesis. By coupling cellular and molecular analyses of a conditional knockout mouse that lacks Elp3 expression in newborn cortical interneurons, we showed that Elongator promotes the migration of interneurons in the forebrain. It cell-autonomously controls the somal and growth cone distribution of actomyosin, as well as the turnover of actin filaments through regulation of cofilin activity. Elongator expression is, thus, required for the dynamic cell shape remodeling that supports tangential migration of cortical interneurons in the forebrain of mouse embryos.

\section{Results}

Conditional deletion of Elp3 in newborn cortical interneurons disrupts their migration

The Elongator complex is expressed in the developing cortical wall where it regulates the generation [29], the migration and the branching of projection neurons [15]. Moreover, we detected Elp3 mRNAs ventrally, in the MGEs of E12.5 embryos with a positive expression gradient (inset of Figure 1A) from the ventricular zone to the mantle zone (MZ) (Figure 1A and 1B). The ventral gradient of Elp3 expression at E12.5 suggested a predominant activity of Elongator in newborn cortical interneurons (inset of Figure 1A). Expression of Elp3 mRNAs progressively dimmed in the ventral progenitor regions of GE with embryonic development (Supplementary information, Figure S1A). To further address the function of Elongator in these cells, we engineered a conditional knockout mouse line $\left(\mathrm{Elp} 3^{\text {lox/lox }}\right)$. Its breeding with Dlx5,6:Cre-GFP transgenic mice [35] resulted in the genetic deletion of Elp3 in newborn cortical interneurons (Elp3cKO; Figure $1 \mathrm{C})$. We validated the deletion of Elp3 expression in cortical interneurons by qRT-PCR on FACS-purified $\mathrm{GFP}^{+}$interneurons dissociated from the forebrain of E14.5 Elp3cKO embryos (Supplementary information, Figure S1B). These data were confirmed by western blot performed on microdissected MGEs from E14.5 embryos (Figure 1D) and on FACS-purified GFP-expressing interneurons from the forebrain of E14.5 embryos (Supplementary information, Figure S1C). The genetic deletion of Elp3 also impairs Elp1 expression (Supplementary information, Figure S1C), as previously reported [29]. We further confirmed the loss of Elp3 expression by immunolabelings of coronal sections from Elp3cKO and WT E12.5 embryos (Figure 1E). Although there were no patterning (Supplementary information, Figure S1D), survival (Supplementary information, Figure S1E and S1F) or proliferation (Supplementary information, Figure S1G and S1H) defects in the GEs of Elp3cKO embryos, we observed a reduced number of migrating Lhx6-expressing interneurons in the developing cortical wall of E12.5 Elp3cKO embryos (Figure $1 \mathrm{~F})$. To test whether Elp3 was required for tangential migration of interneurons, we quantified the number of GFP-expressing interneurons crossing the cortico-striatal boundary (CSB, Figure $1 \mathrm{G})$ and entering into the cortical wall at E12.5 (Figure 1H and 1I) and E14.5 (Figure 1J). The subventricular zone and MZ streams were significantly affected at E14.5 (Figure 1K and 1L). Although the migration of GFP-expressing interneurons was reduced at both stages of development, this phenotype was not observed at later embryonic stage (Supplementary information, Figure S1I). Altogether, these results demonstrate the requirement of Elongator for proper tangential migration of interneurons in the developing cerebral cortex.

Loss of Elp3 impairs nucleokinesis and dynamic branching of migrating interneurons

Cortical interneurons migrate through repetitive cycles of saltatory nuclear movements (nucleokinesis) and dynamic branching of the leading process [10]. Hence, we performed live imaging experiments to investigate the cellular defects resulting from Elp3 deletion. We recorded the migration of GFP-expressing interneurons either exiting the MGE explants or moving within organotypic forebrain cultures [11]. MGE explants were isolated from Elp3cKO or WT E13.5 embryos and cultured onto a monolayer of WT homochronic-mixed cortical culture $24 \mathrm{~h}$ before the recordings were initiated (Figure 2A). Despite their ability to migrate out of the explants, Elp3cKO interneurons had both reduced velocity (Figure $2 \mathrm{~B}$ ) and directional persistence (Figure 2C-2E) as compared with control WT interneurons. Quantitative analysis revealed a reduced percentage of Elp3cKO interneurons with a swelling ahead of the nucleus (initiation 
A

Elp3 mRNA AS probe
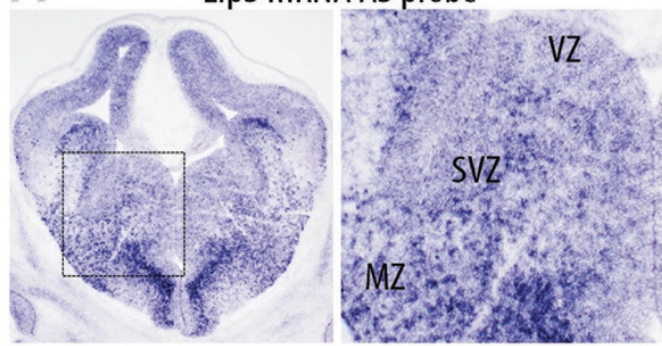

B Elp3 mRNA S probe

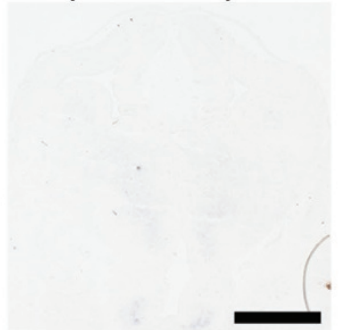

C

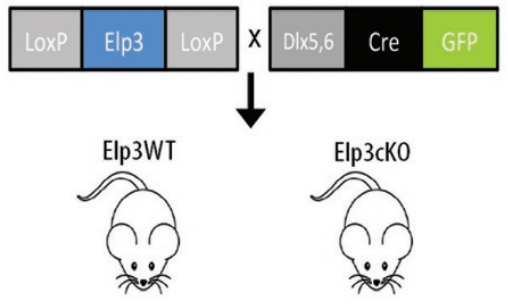

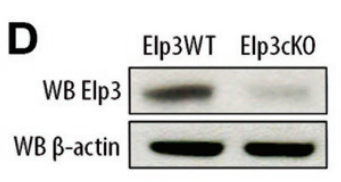

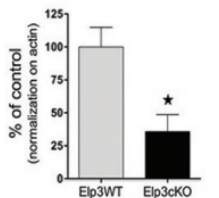

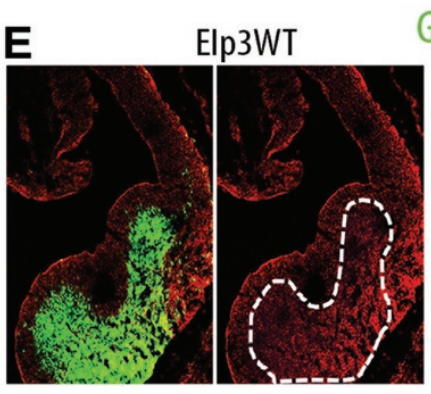

GFP/Elp3
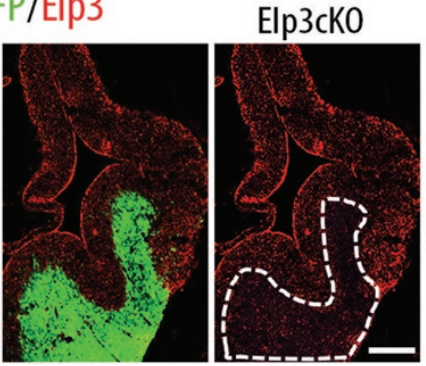

GFP/ßIII-tubulin/Hoechst
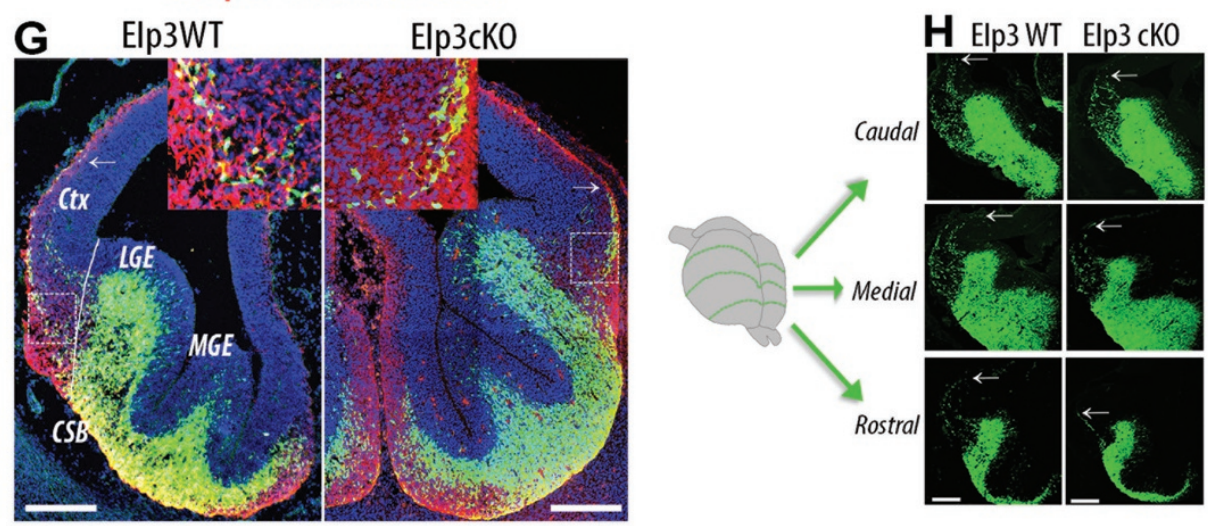

F Lhx6 mRNAs

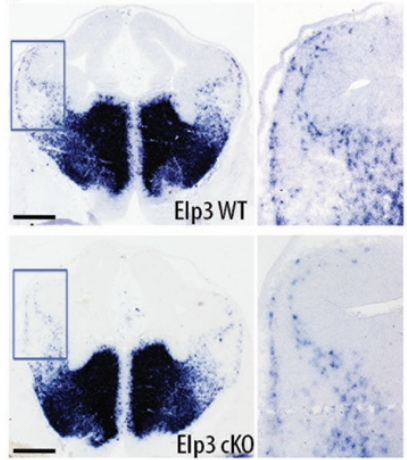

I
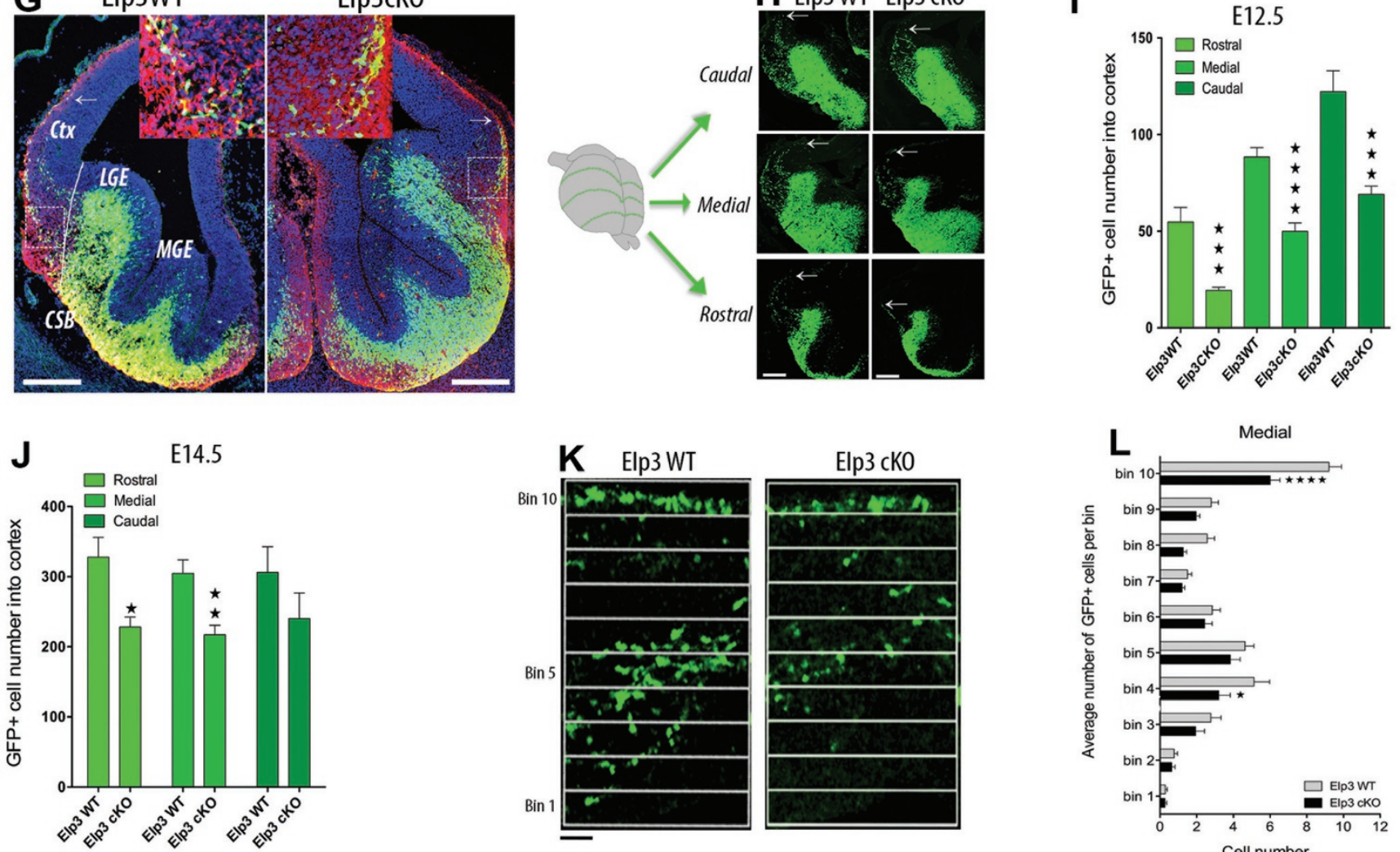
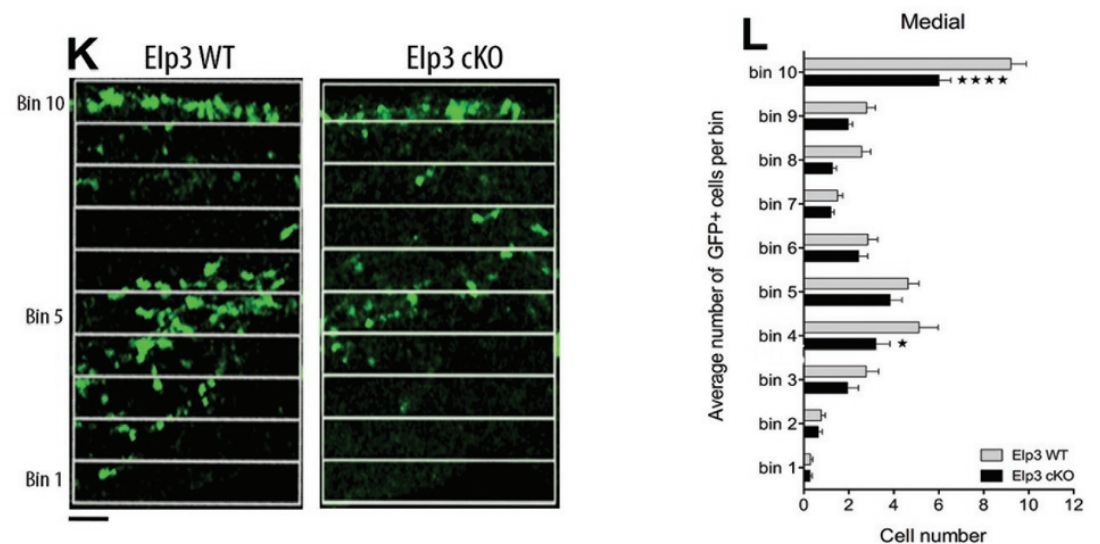

Figure 1 Conditional deletion of Elp3 in interneurons impairs their migration. (A-E) Detection of Elp3 on forebrain sections of E12.5 mouse embryos showing distribution of its mRNAs with antisense (AS; $\mathbf{A}$ ) but not sense probe (S; $\mathbf{B})$. Mouse breeding strategy to generate Elp3cKO embryos (C), validation of the conditional loss of Elp3 in Elp3cKO MGE by western blot (D) and immunolabelings of E12.5 forebrain sections (GFP is green and Elp3 is red; E). (F-L) Tangential migration of cortical 
interneurons. In situ hybridizations on WT E12.5 forebrain sections showing Lhx6-expressing interneurons. The insets show magnified area of the cortical wall invaded by migrating interneurons (blue, F). Immunolabeling of E12.5 forebrain sections from Elp3 WT or Elp3cKO embryos showing expression of GFP (green), Blll-tubulin (red) and Hoechst for nuclei (blue) (G). Immunodetection of GFP-expressing interneurons from Elp3 WT or Elp3cKO embryos that entered into the cortical wall at distinct rostro-caudal levels (H, arrows show front of migration). Interneuron numbers were quantified at E12.5 (I; rostral: 54.8 \pm 7.4 cells for WT and $19.4 \pm 1.6$ cells for Elp3cKO, ${ }^{* \star *} P<0.0002$, $t$-test; medial: $88.3 \pm 4.9$ for WT and $49.9 \pm 4.3$ cells for Elp3cKO, ${ }^{* * *} P<0.0001, t$-test; caudal: $122.2 \pm 10.8$ cells for WT and $69.1 \pm 4.2$ cells for Elp3cKO, ${ }^{* * *} P<0.0002, t$-test; $n=$ 3 brains per condition) or E14.5 ( $\mathrm{J}$; rostral: $327.6 \pm 28.2$ cells for WT and $227.9 \pm 14.24$ cells for Elp3cKO, ${ }^{*} P<0.102, t$-test; medial: $304.8 \pm 19.1$ cells for WT and $217.1 \pm 13.7$ cells for Elp3cKO, ${ }^{* *} P<0.0039$, $t$-test; caudal: $306.2 \pm 39.5$ cells for WT and $240.3 \pm 36.5$ cells for Elp3cKO, ns, $t$-test; $n=6$ brains per condition). Laminar distribution of interneurons in the cortical wall (medial level) of E14.5 Elp3 WT or Elp3cKO embryos (K) quantified in bins (L; Bin 10, $9.2 \pm 0.7$ cells for WT and $6.0 \pm$ 0.5 cells for Elp3cKO, ${ }^{* * * *} P<0.0001$; Bin $4,5.1 \pm 0.9$ cells for WT and $3.2 \pm 0.6$ cells for Elp3cKO, ${ }^{*} P<0.05 ;{ }^{* * * \star} P<0.0001$ (interaction) two ways ANOVA and Bonferroni's post hoc test; $n=6$ brains per condition). Abbreviations, cortex (Ctx), cortico-striatal boundary (CSB), lateral ganglionic eminence (LGE), medial ganglionic eminence (MGE), cortical plate (CP), mantle zone (MZ), ventricular/subventricular zone (VZ/SVZ).Scale bars represent $100 \mu \mathrm{m}$ (B, E, F, G, H) or $25 \mu \mathrm{m}$ (K; See also Supplementary information, Figure S1). CP, cortical plate; CSB, cortico-striatal boundary; Ctx, cortex; LGE, lateral ganglionic eminence; MGE, medial ganglionic eminence; MZ, mantle zone; SVZ, subventricular zones; VZ, ventricular zones.

of nucleokinesis, Figure $2 \mathrm{~F}$ ). The maximal swelling area was not different between genotypes (data not shown). Moreover, we observed less frequent and shorter nuclear translocations (Figure $2 \mathrm{G}$ and $2 \mathrm{H}$ ). The dynamic branching of the leading process was also impaired upon loss of Elp3. This was exemplified by the reduced frequency of growth cone splitting, which underlies the formation of new branches (Figure 2I). The average length of the leading process was also shorter in Elp3cKO interneurons (Figure 2J).

We next validated these results in the developing cortex by performing live imaging of organotypic cultures from E12.5 WT or Elp3cKO forebrains that were maintained $24 \mathrm{~h}$ in vitro [11]. The recordings were acquired within the cortical wall, just above the CSB (region squared in Figure 2K). The resolution of this experimental setting allowed us to assess cell migration (including nucleokinesis) but not branching dynamics. We confirmed that Elp3cKO interneurons had both reduced velocity (Figure 2L) and migration persistence (Figure $2 \mathrm{M}$ ) while navigating in the cortical wall, as compared with control interneurons (Supplementary information, Movies S1 and S2). The nucleokinesis process was also impaired (Figure $2 \mathrm{~N}$ and 2O), confirming the results obtained with Elp3cKO MGE explants. We next performed rescue experiments with Elp3 or its point mutant Y529A (Elp3mKAT, Supplementary information, Figure S2A) that retains very-low residual KAT activity [36] (Figure $2 \mathrm{P}$ and 2Q). Focal electroporation of Elp3 in Elp3cKO interneurons did not increase the persistence of migration (Figure 2R) but significantly raised the velocity (Figure $2 \mathrm{~S})$, nucleokinesis amplitude and frequency, as compared with control vectors in Elp3cKO (Figure 2T and 2U). Surprisingly, the expression of Elp3mKAT also improved most migration parameters, suggesting that the contri- bution of Elp3 to the migration of interneurons does not predominantly require its acetyltransferase activity (Figure 2Q-2U). Accordingly, the acetylation of MTs, a posttranslational modification promoted by Elongator in cortical projection neurons to support their migration [15], was not impaired in Elp3cKO interneurons (Supplementary information, Figure S2B). In addition, the loss of Elp3 acetylase activity can reduce cortical neurogenesis by inducing endoplasmic reticulum (ER) stress and activating the PERK branch of the unfolded protein response (UPR) pathway [29]. Although activation of the UPR can impair cell migration [37], this was not responsible for the migration phenotype of Elp3cKO interneurons, as supported by the physiological expression level of two downstream targets of the PERK pathway (ATF4 and $S L C 7 a 3$ ) in FACS-purified Elp3cKO interneurons (Supplementary information, Figure S2C and S2D).

Interneurons that lack Elp3 display abnormal actomyosin dynamics during migration

As proper regulation of actomyosin contractions is critical for nucleokinesis and growth cone splitting [10, $11]$, we investigated whether the migration phenotype of Elp3cKO interneurons might result from dynamic defects in actomyosin. Western blot performed on microdissected MGEs of E14.5 WT or Elp3cKO embryos revealed an increased phosphorylation of the regulatory light chain of the myosin II (pMLC), which is a readout of myosin II activity [11], in Elp3cKO MGE (Figure $3 \mathrm{~A})$. There was also greater recruitment of MLC to actin filaments (F-actin) upon Elp3 depletion (Supplementary information, Figure S4P), which could suggest an overall increase of actomyosin activity in Elp3cKO interneurons. Accordingly, protein levels of total MLC kinase (MLCK) [38] and its inactivated form (pMLCK) (Figure 

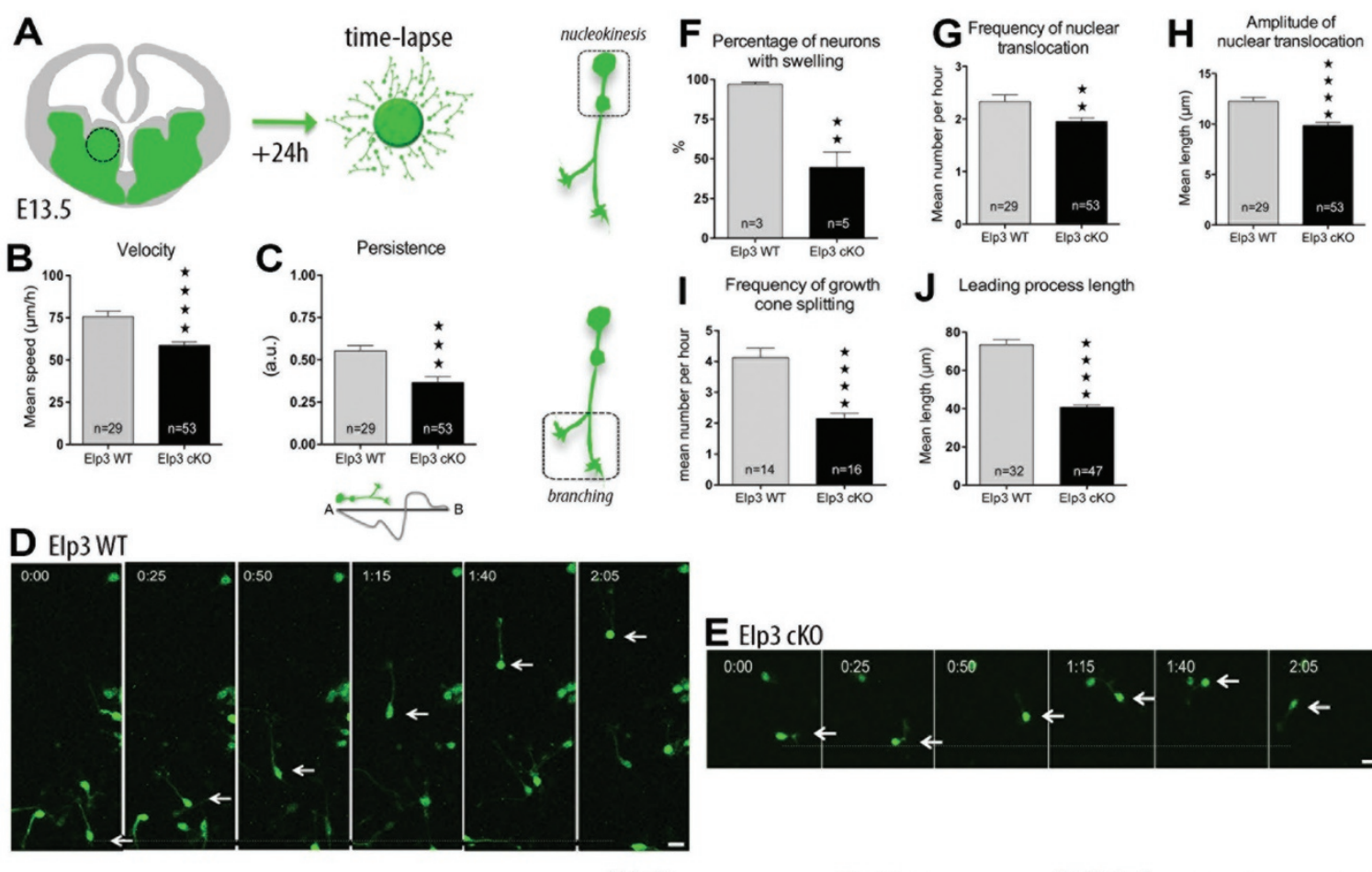

J Leading process length
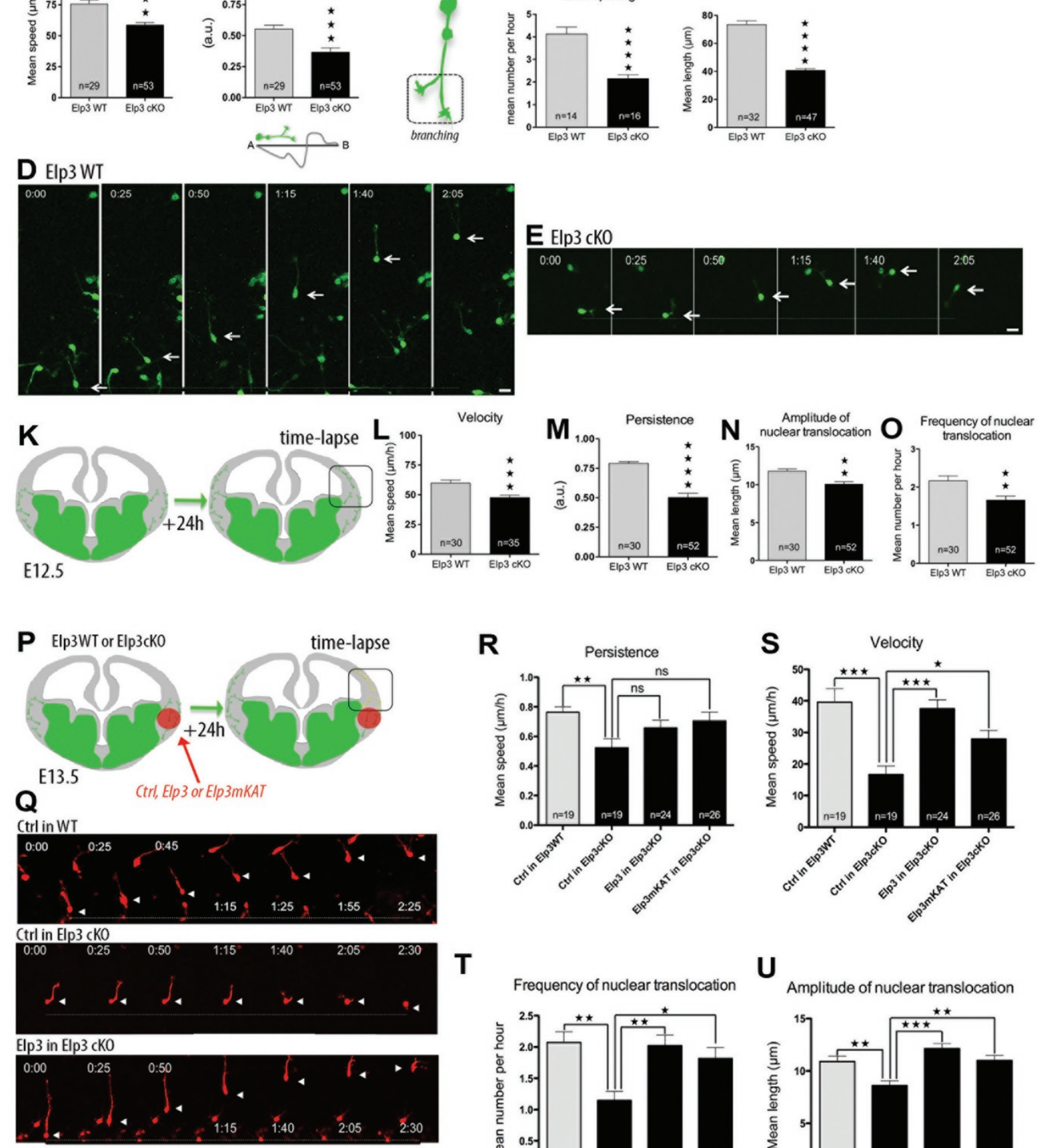

$\mathbf{T}$

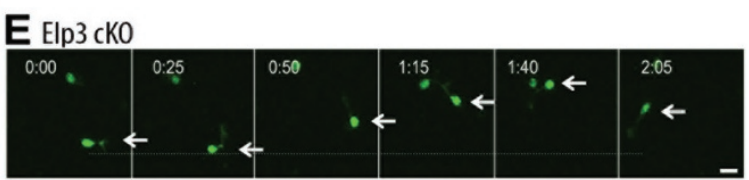

Elp3 mKAT in Elp3 cKO
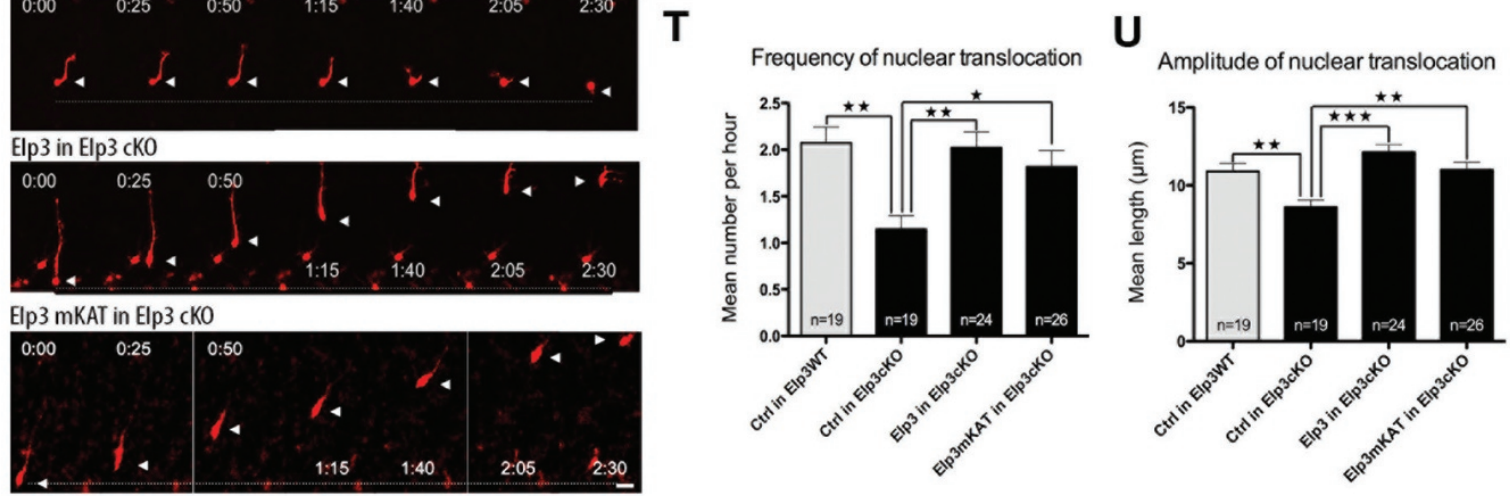
Figure 2 Elp3 expression is required for proper nucleokinesis and leading process branching. (A-E) Cell migration defects in cultured interneurons upon deletion of Elp3. Scheme of the MGE explant migration assay (A). Histograms of the velocity (B; $75.6 \% \pm 3.2 \%$ for WT and $58.6 \% \pm 2.2 \%$ for Elp3cKO, ${ }^{* * *} P<0.0001, t$-test; $n=29-53$ cells per condition in three independent experiments) and the migration persistence (C; $0.55 \pm 0.03$ for WT and $0.36 \pm 0.03$ for Elp3cKO, ${ }^{* * *} P<0.0005, t$-test; $n$ = 29-53 cells per condition in three independent experiments) of cortical interneurons (genotypes as indicated). Time lapse sequences showing the migration of (white arrows on D and E) an Elp3WT interneuron (D) or an Elp3cKO interneuron (E). (F-J) Histograms of the percentage of neurons with swelling (F; 96.9\% $\pm 1.4 \%$ for WT and $44.6 \% \pm 9.7 \%$ for Elp3cKO, ${ }^{* *} P<$ $0.0069, t$-test; $n=3-5$ brains per condition), the frequency of nuclear translocation of migrating interneurons $(\mathbf{G} ; 2.3 \% \pm 0.1 \%$ for WT and $1.9 \% \pm 0.1 \%$ for Elp3cKO, ${ }^{* * *} P<0.0070, t$-test; $n=29-53$ cells per condition in three independent experiments), the amplitude of nuclear translocation $\left(\mathbf{H} ; 12.3 \pm 0.4\right.$ for WT and $9.9 \pm 0.3$ for Elp3cKO, ${ }^{* * * *} P<0.0001, t$-test; $n=29-53$ cells per condition in three independent experiments), the frequency of growth cone splitting (I; $4.1 \pm 0.3$ for WT and $2.1 \pm 0.2$ for Elp3cKO, ${ }^{* * \star} P<0.001, t$-test; $n=14-16$ cells per condition in three independent experiments) and the leading process length ( $\mathbf{J} ; 73.4 \pm 2.7$ for WT and $40.7 \pm 1.3$ for Elp3cKO, ${ }^{* * * *} P<0.0001, t$-test; $n=29-53$ cells per condition in three independent experiments) of migrating interneurons, genotype, as indicated. (K-O) Interneuron migration defects in organotypic culture of E12.5 forebrain slice. Experimental up to track interneuron migration in cultured forebrain slice (K). Histograms of the migration velocity (L; $59.8 \pm 2.7$ for WT and $47.6 \% \pm 2.0 \%$ for Elp3cKO, ${ }^{* * *} P<0.0006, t$-test; $n=30$-35 cells per condition in three independent experiments), the migration persistence (M; $0.79 \pm 0.02$ for WT and $0.50 \pm 0.03$ for Elp3cKO, ${ }^{* * \star *} P<0.0001$, $t$-test; $n=30-52$ cells per condition in three independent experiments), the amplitude of nuclear translocation ( $\mathbf{N} ; 11.8 \pm 0.3$ for WT and $10.1 \pm 0.3$ for Elp3cKO, ${ }^{* *} P<0.0015, t$-test; $n=30$-35 cells per condition in three independent experiments), the frequency of nuclear translocation $\left(0 ; 2.2 \pm 0.1\right.$ for WT and $1.7 \pm 0.2$ for Elp3cKO, ${ }^{* *} P<0.0044, t$-test; $n=30$-35 cells per condition in three independent experiments) of migrating cortical interneurons, genotypes as indicated. (P-U) Rescue experiments of the migration defect resulting from loss of Elp3 expression. Scheme showing the experimental procedure to rescue the migration defects (P). Time-lapse sequences showing the migration of (white arrowheads) Elp3WT or Elp3cKO interneurons in MGE electroporated with distinct plasmid constructs as indicated (Q). Histograms of the migration persistence (R; 0.76 \pm 0.03 for ctrl in Elp3WT, $0.52 \pm 0.06$ for ctrl in Elp3cKO, $0.6576 \pm 0.05$ for Elp3 in Elp3cKO and $0.70 \pm 0.05$ for Elp3mKAT in Elp3cKO, ${ }^{*} P<0.0103$, one way ANOVA and Dunnett's post hoc test; $n=19-26$ cells in three independent experiments), the migration velocity $(\mathbf{S} ; 39.61 \pm 4.27$ for ctrl in Elp3WT, $16.67 \pm 2.77$ for ctrl in Elp3cKO, $37.55 \pm 2.78$ for Elp3 in Elp3cKO and $27.93 \pm 2.75$ for Elp3mKAT in Elp3cKO, ${ }^{* *} P<0.001$, one way ANOVA and Dunnett's post hoc test; $n=19-26$ cells in three independent experiments), the frequency of nuclear translocation ( $T ; 2.07 \pm 0.16$ for ctrl in Elp3WT, $1.14 \pm 0.14$ for ctrl in Elp3cKO, $2.022 \pm 0.16$ for Elp3 in Elp3cKO and $1.81 \pm 0.17$ for Elp3mKAT in Elp3cKO, ${ }^{* *} P<0.0021$, one way ANOVA and Dunnett's post hoc test; $n=19-26$ cells in three independent experiments), the amplitude of nuclear translocation (U; $10.90 \pm$ 0.50 for ctrl in Elp3WT, $8.613 \pm 0.45$ for ctrl in Elp3cKO, $12.15 \pm 0.45$ for Elp3 in Elp3cKO and $11.02 \pm 0.45$ for Elp3mKAT in Elp3cKO. ${ }^{* \star *} P<0.0001$, one way ANOVA and Dunnett's post test; $n=19-26$ cells in three independent experiments) of cortical interneurons, genotypes as indicated. Scale bars represent $15 \mu \mathrm{m}$ (D, E, Q). See also Supplementary information, Figure S2.

3B) were increased in MGE from E14.5 Elp3cKO embryos as compared with control embryos. The accumulation of pMLCK without change of the pMLCK/MLCK ratio between genotypes (Figure $3 \mathrm{~B}$ ) most likely resulted from the increased transcription of $M L C K$ mRNAs (Figure $3 \mathrm{C}$ ) rather than from modulation of its activation through the Rac or the RhoA pathways (Supplementary information, Figure S4A-S4I). Therefore, we reasoned that targeting MLCK activity with its blocker ML-7 would reduce pMLC and, thus, rescue the migration defects of Elp3cKO interneurons. To test this hypothesis, we performed time-lapse recordings on cultured MGE explants from E13.5 WT or Elp3cKO embryos in the presence of ML-7 or DMSO (vehicle) (Figure 3D). Surprisingly, reducing the overall phosphorylation of MLC (Supplementary information, Figure S2E), hence myosin II activity, improved neither the velocity nor the migration persistence of Elp3cKO interneurons (Figure 3E$3 \mathrm{~F})$. In these conditions, it is noteworthy that addition of the vehicle slightly affected the migration persistence of neurons from both genotypes (compare Figure 2C and Figure $3 \mathrm{~F}$ ). Moreover, both nuclear translocation (Figure $3 \mathrm{G}$ and $3 \mathrm{H}$ ) and branching parameters (Figure 3I and $3 \mathrm{~J}$ ) remained significantly impaired upon loss of Elp3 expression in migrating interneurons, irrespective of treatment with ML-7.

To further explore the link between Elongator and actomyosin, we assessed the dynamic distribution of actomyosin contractions by electroporating MGE explants with LifeAct constructs. We monitored LifeAct signal, which reflects the actin microfilament (F-actin) condensation associated with actomyosin contractions in migrating interneurons [11, 39] (Figure 3K, 3L and Supplementary information, Movies S3 and S4). Elp3cKO interneurons showed a significant increase in growth cone surface before splitting (Figure $3 \mathrm{M}$ ) with reduced actomyosin intensity per unit of growth cone surface, as compared with control (Figure $3 \mathrm{~N}$ ). Such a defect 

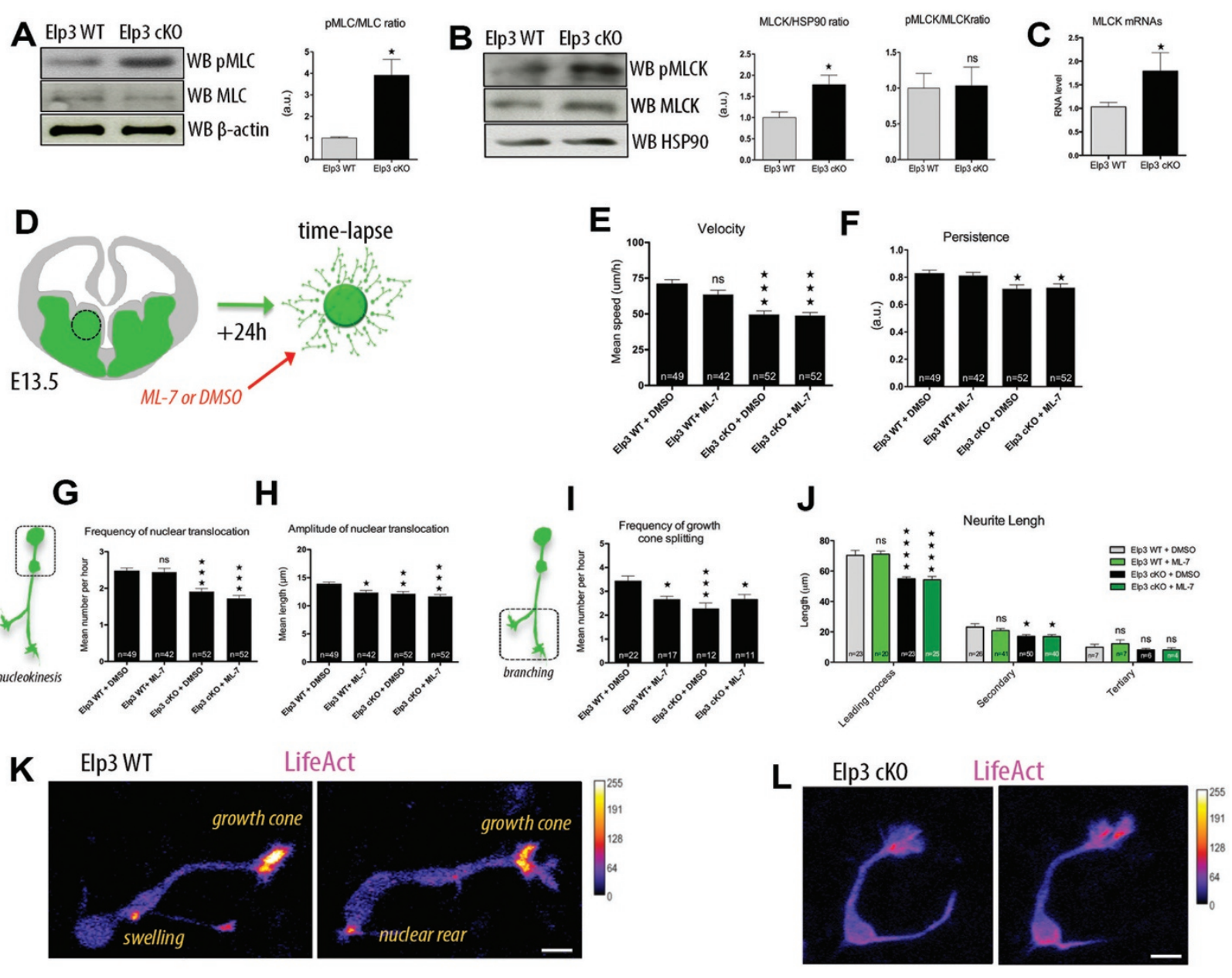

M
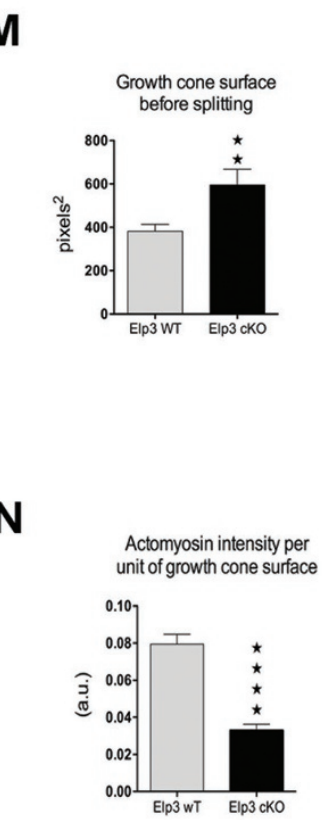

0
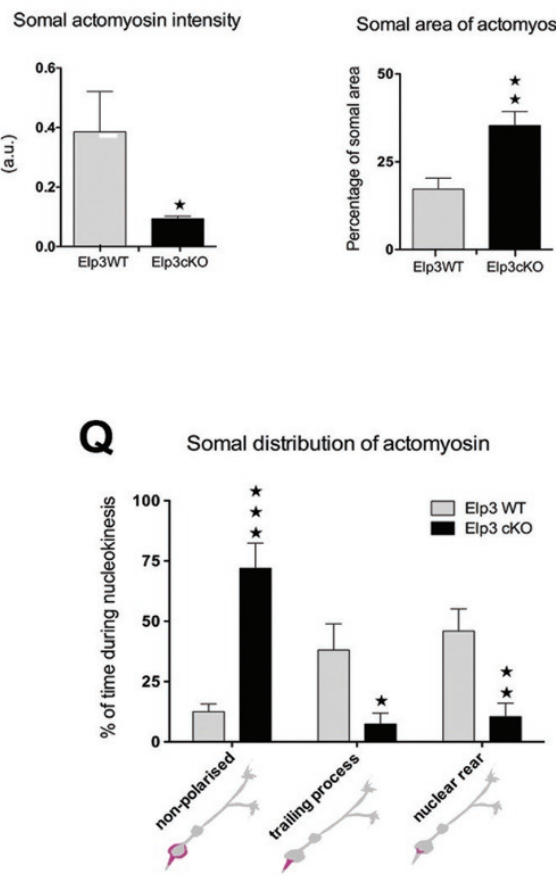

P

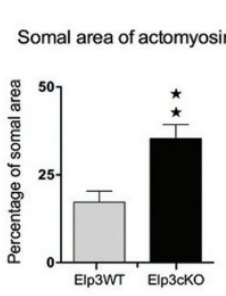

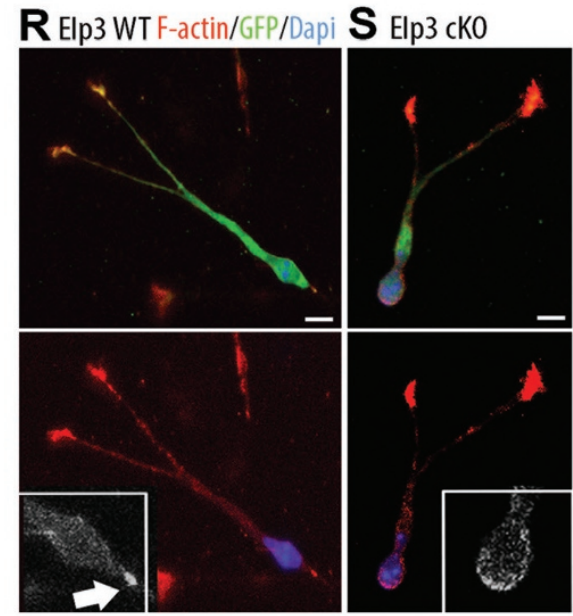

T Elp3 WT F-actin U Elp3 ck0

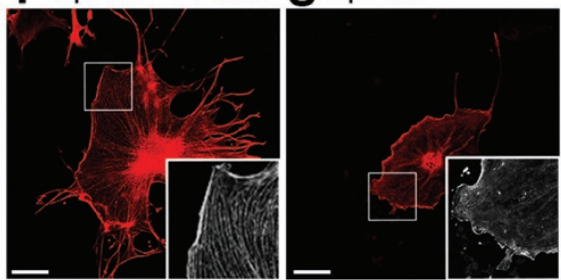


Figure 3 Loss of Elp3 impairs the intensity and the subcellular distribution of actomyosin forces in migrating interneurons. (AJ) Disregulation of actomyosin contractility upon Elp3 deletion. Expression of MLC and pMLC (A; $1.0 \pm 0.1$ for WT and $3.9 \pm$ 0.7 for Elp3cKO, ${ }^{*} P<0.0167, t$-test; $n=3$ MGE extracts per condition) or MLCK proteins (B; MLCK/HSP90 ratio: $1.0 \pm 0.1$ for WT and $1.8 \pm 0.2$ for Elp3cKO, ${ }^{*} P<0.0403$, $t$-test; MLCK/pMLCK ratio: $1.0 \pm 0.2$ for WT and $1.0 \pm 0.3$ for Elp3cKO, ns, $t$-test; $n=3 \mathrm{MGE}$ extracts per condition) monitored by western blot or MLCK mRNAs by qRT-PCR (C; $1.0 \pm 0.1$ for WT and $1.8 \pm 0.4$ for Elp3cKO, * $P<0.0351, t$-test; $n=3$ MGE extracts per condition) in Elp3WT or Elp3cKO MGE extracts from E14.5 embryos. Scheme of the MGE explant migration assay with drug treatment for live imaging (D). Histograms showing the migration velocity ( $\mathrm{E} ;{ }^{* * * *} P<0.0001$, one way ANOVA and Tukey's post hoc test; $n=49-52$ cells in three independent experiments), the migration persistence ( $\mathbf{F} ;{ }^{* *} P<0.0020$, one way ANOVA and Tukey's post hoc test; $n=49-52$ cells in three independent experiments), the frequency of nuclear translocation of migrating interneurons (G; ${ }^{* * * *} P<0.0001$, one way ANOVA and Tukey's post hoc test; $n=49-52$ cells in three independent experiments), the amplitude of nuclear translocation $\left(\mathbf{H} ;{ }^{* * *} P<0.0001\right.$, one way ANOVA and Tukey's post hoc test; $n=49-52$ cells in three independent experiments), the frequency of growth cone splitting (I; ${ }^{* *} P<0.0011$, one way ANOVA and Tukey's post hoc test; $n=11-22$ cells in three independent experiments) and the leading process and secondary and tertiary neurite length $\left(\mathbf{J}\right.$; ${ }^{* * \star} P<0.0001,{ }^{*} P<0.0050$, one way ANOVA and Tukey's post hoc test; $n=49-52$ cells in three independent experiments) of migrating interneurons, genotype and drug treatment as indicated. (K-Q) Assessment of distribution and intensity of actomyosin contractions in Elp3WT or Elp3cKO cortical interneurons. Time-lapse sequences showing the distribution of condensated F-actin (fire) in an Elp3WT (K) or an Elp3cKO (L) interneuron after electroporation with LifeAct construct. Histograms of the growth cone surface before splitting (M; $382.6 \pm 31.8$ for WT and $594.7 \pm 73.2$ for Elp3cKO, ${ }^{* *} P<0.0090, t$-test; $n=20-22$ cells per condition in three independent experiments), the actomyosin intensity per unit of growth cone surface $\left(\mathbf{N} ; 0.08 \pm 0.1\right.$ for WT and $0.03 \pm 0.01$ for Elp3cKO, ${ }^{* * * *} P<0.0001$, $t$-test; $n=20$-22 cells per condition in three independent experiments), the somal actomyosin intensity (0; $0.39 \pm 0.1$ for WT and $0.1 \pm 0.01$ for Elp3cKO, ${ }^{*} P<0.0489, t$-test; $n=8$ cells per condition in three independent experiments), the somal area of actomyosin $\left(\mathbf{P} ; 17.2 \pm 3.1\right.$ for WT and $35.3 \pm 3.9$ for Elp3cKO, ${ }^{* *} P<0.0032$, $t$-test; $n=8$ cells per condition in three independent experiments), the somal distribution of actomyosin $\left(\mathbf{Q} ;{ }^{* * * *} P<0.0001\right.$, two way ANOVA and Bonferroni's post hoc test; $n=9-10$ cells in three independent experiments). (R-U) Subcellular distribution of F-actin (red) in cortical interneurons (green, cytoplasmic GFP; blue, Dapi-counterstained nuclei) (R, S) or MEFs (T, U). Scale bars represent $10 \mu \mathrm{m}$ (K, L, R, S, T, U). See also Supplementary information, Figure S2.

suggested a reduced efficiency of actomyosin forces to promote growth cone splitting in Elp3cKO neurons (Figure 2I). Actomyosin contractions are polarized behind the nucleus of migrating cortical interneurons in order to propel the nucleus forward [10]. Therefore, we analyzed the somal distribution of LifeAct signals during the migration process. Our time-lapse analysis showed that the LifeAct signal was overall less intense and more widely dispersed around the nucleus (cup-like shape) of Elp$3 \mathrm{cKO}$ interneurons compared with controls, suggesting a redistribution of actomyosin forces during migration (Figure 3K, 3L, 3O and 3P). Live imaging of migrating neurons revealed a long-lasting accumulation of non-polarized distribution of LifeAct around the nucleus of Elp3cKO interneurons (Figure 3Q). This defect was mirrored by the disrupted somal distribution of F-actin (Figure 3R and 3S), the substrate of myosin II motors [11], upon loss of Elp3 expression. Proper regulation of actomyosin dynamics requires a F-actin network whose formation was disrupted in Elp3 $\mathrm{KO}$ mouse embryonic fibroblasts (MEFs), as compared with their controls (Figure 3T and 3U). Knockdown of Elp1 expression has previously been associated with expression defects of Filamin A (FlnA) in cultured cells [40], an actin-binding protein that crosslinks $\mathrm{F}$-actin in orthogonal networks within the cytoplasm [41]. Despite being a strong can- didate, our analyses revealed no difference in either the protein level (Supplementary information, Figure S2F) or the subcellular distribution (Supplementary information, Figure S2G) of FlnA in cortical interneurons from both genotypes. Accordingly, we postulated that the migration phenotype of Elp3cKO interneurons might predominantly arise from impaired actin cytoskeleton formation that would secondarily impact actomyosin dynamics.

\section{The genetic deletion of Elp3 results in increased actin severing by cofilin in cortical interneurons}

The disruption of the actin cytoskeleton was supported by the existence of an increased G-(globular)/F-actin ratio in MGE extracts from Elp3cKO E14.5 embryos, as compared with control (Figure 4A). Interestingly, Neuroblastoma 2A (N2A) cells knocked down for Elp3 showed a migration delay in wound-healing assays as compared with controls (Supplementary information, Figure S3A and $\mathrm{S} 3 \mathrm{~B}$ ) that correlates with an impaired $\mathrm{G} / \mathrm{F}$-actin ratio (Supplementary information, Figure S3C). To visualize the different cellular compartments in interneurons, we performed immunolabelings to detect G- and F-actin (Figure 4B) and to measure the G/F-actin intensity profile. These experiments revealed a significant change of $\mathrm{G} / \mathrm{F}$-actin proportion in cell body, branches and growth cone of Elp3cKO interneurons, as compared with con- 


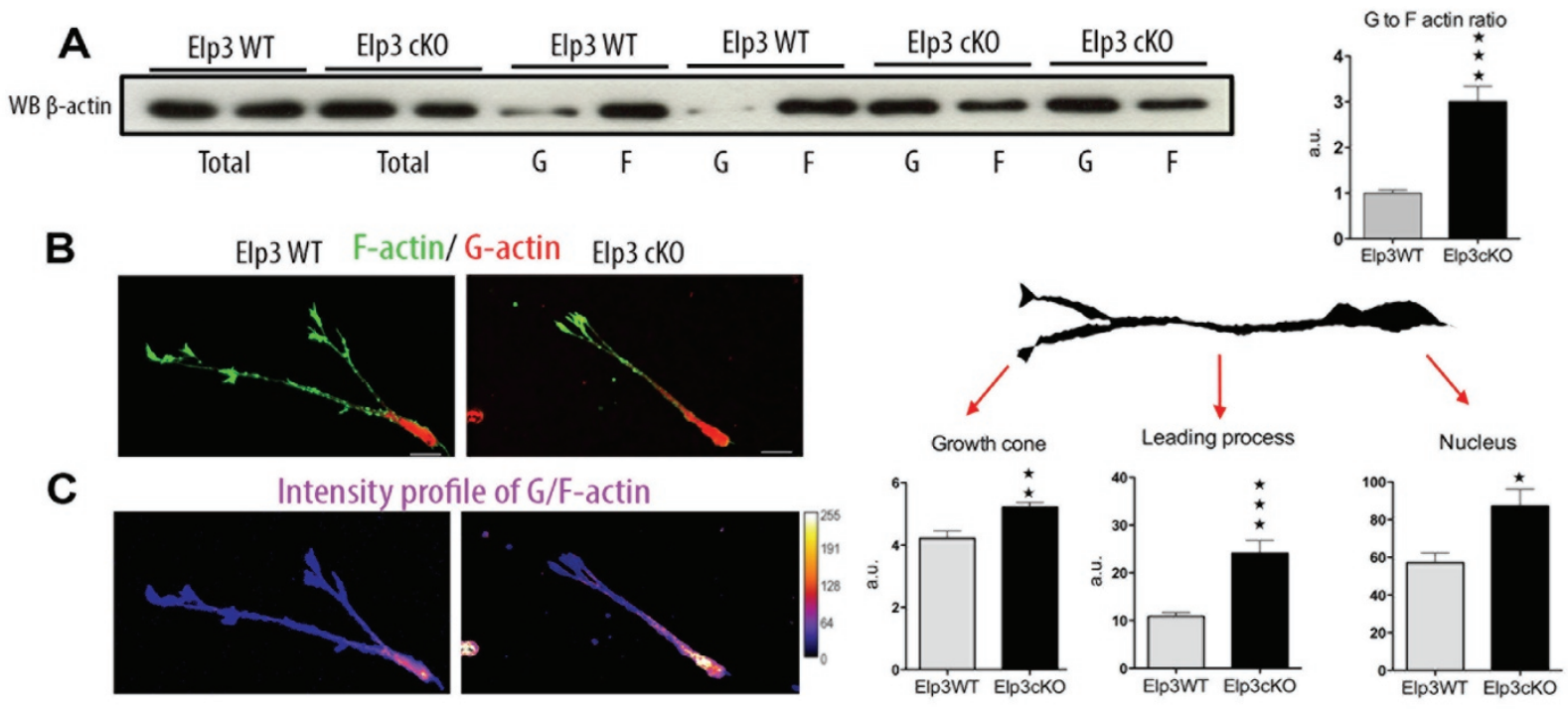

D Elp3WT Elp3 cko
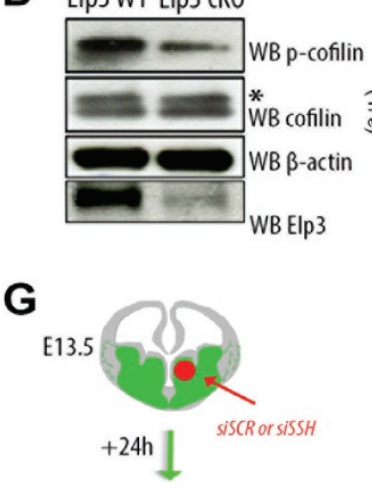

time-lapse

J Frequency of nuclear translocation

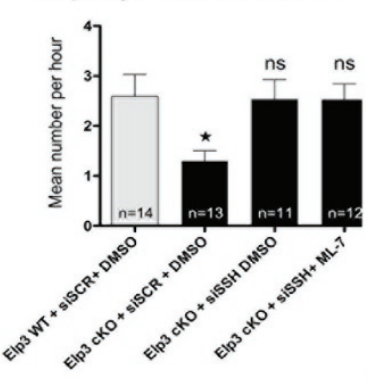

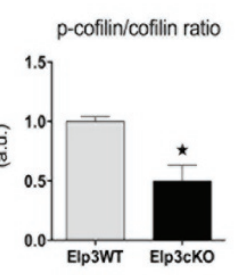

E Input SN IP
IPFlag +++

IP Elp3

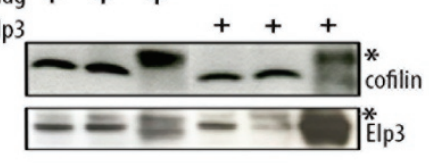

F Input SN IP SN IP

$\mathrm{IPCofilin}+++$ IPHA

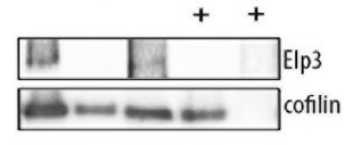

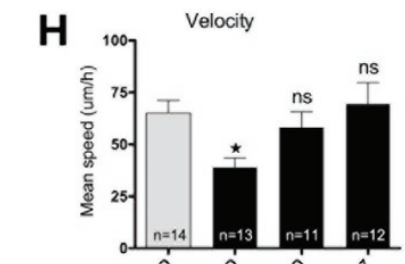

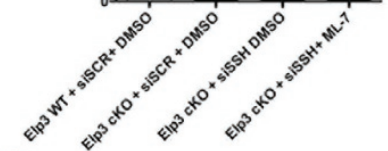

$\mathbf{K}_{\text {Amplitude of nuclear translocation }}$

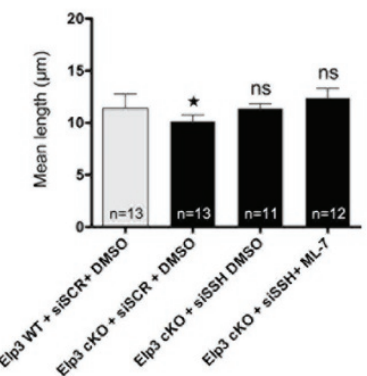

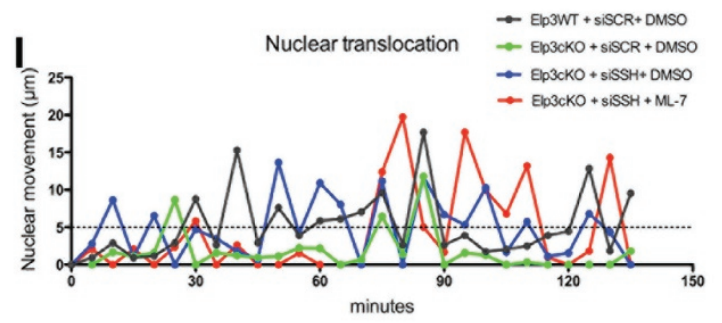

L Frequency of growth

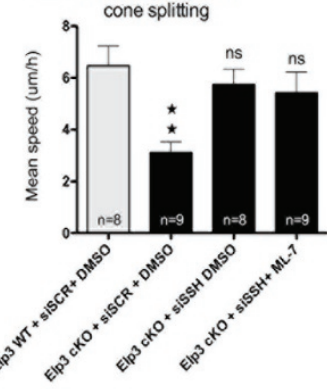

M Growth cone surface

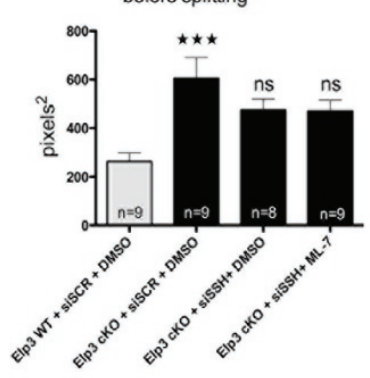

$\mathbf{N}$

Mean length of neurites

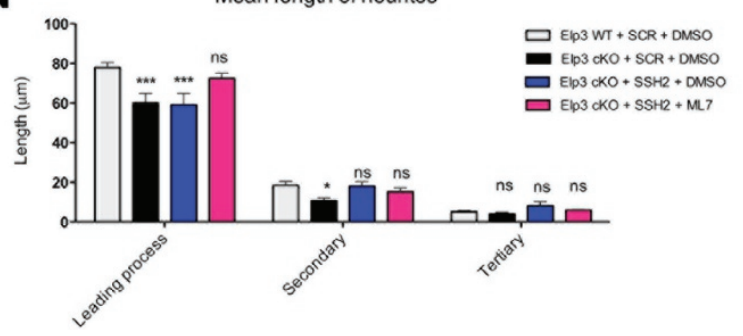


Figure 4 Deregulation of cofilin activity upon Elp3 depletion is responsible for interneuron migration defects. (A-F) Change of F-actin severing by cofilin upon Elp3 deletion. Western blot of G- and F-actin fractions in MGE extracts from E14.5 Elp3WT or Elp3cKO embryos (A) and $\mathrm{G}$ to $\mathrm{F}$ ratio quantification ( $1.00 \pm 0.02$ for WT and $0.37 \pm 0.04$ for Elp3cKO, ${ }^{* * *} P<0.0002, t$-test; $n=4$ brains per genotype). Immunolabelings for F-actin (phalloidin, green) and G-actin (anti-deoxyribonuclease I (DNAse I), red) in cortical interneurons migrating in matrigel (B), intensity profiles of G/F-actin (fire) measured at different parts of the cell body (C, growth cone: $4.21 \pm 0.23$ for WT and $5.22 \pm 0.15$ for Elp3cKO, ${ }^{* *} P<0.0027$, $t$-test; leading process: $10.91 \pm$ 0.77 for WT and $24.08 \pm 2.73$ for Elp3cKO, ${ }^{* * *} P<0.0005$, $t$-test; nucleus: $57.09 \pm 5.22$ for WT and $87.18 \pm 8.95$ for Elp3cKO, ${ }^{*} P<0.0333$, $t$-test; $n=11$ for Elp3WT and $n=21$ for Elp3cKO interneurons for all measurements). Expression level of cofilin and its phosphorylated form (p-cofilin) in MGE extracts from E14.5 Elp3WT or Elp3cKO embryos quantified as ratio of p-cofilin over cofilin expression (D; $1.00 \pm 0.04$ for WT and $0.37 \pm 0.04$ for Elp3cKO, ${ }^{*} P<0.0114, t$-test; $n=4$ independent experiments). Immunoprecipitation of cofilin or Elp3 by anti-Elp3 or anti-cofilin antibodies, respectively, in MGE extracts from E14.5 Elp3WT embryos (E, F). (G-N) Cell migration rescue in cultured interneurons upon deletion of Elp3 and SSH2 with or without ML-7 treatment. Scheme of the MGE explant migration assay with siRNA expression and drug treatment for live imaging (G). Histogram showing the migration velocity $\left(\mathbf{H} ;{ }^{*} P<0.0121\right.$, one way ANOVA and Dunnett's post test; $n=11-14$ cells in three independent experiments). Histogram showing nuclear displacements during migration per unit of time for Elp3WT or Elp3cKO interneurons treated by drugs and/or siRNA, as indicated on the graph. Only nuclear movements above $5 \mu \mathrm{m}$ (black dotted line) are considered significant (I). Histograms showing the frequency of nuclear translocation $\left(\mathbf{J}\right.$; ${ }^{*} P<0.0116$, one way ANOVA and Dunnett's post test; $n=11-14$ cells in three independent experiments), the amplitude of nuclear translocation (K; ${ }^{*} P<0.0116$, one way ANOVA and Dunnett's post hoc test; $n=11-14$ cells in three independent experiments), the frequency of growth cone splitting $\left(\mathrm{L} ;{ }^{*} P<0.0069\right.$, one way ANOVA and Dunnett's post test; $n=11-14$ cells in three independent experiments), the growth cone surface before splitting $\left(\mathbf{M} ;{ }^{* \star} P<0.018\right.$, one way ANOVA and Dunnett's post hoc test; $n=8-9$ cells in three independent experiments) and the mean length of neurite ( $\mathbf{N}$, leading process: $77.95 \pm 2.56, n=11$ cells for Elp3WT + siSCR + DMSO, $60.162 \pm 4.784, n=11$ cells for Elp3cKO + siSCR + DMSO, $59.13 \pm 5.85, n=8$ cells for Elp$3 \mathrm{cKO}+\mathrm{siSSH}+\mathrm{DMSO}$ and $75.50 \pm 2.64, n=10$ cells for Elp3cKO + siSSH + ML7; secondary: $18.57 \pm 1.83, n=27$ neurites for Elp3WT + siSCR + DMSO, $10.72 \pm 1.52, n=25$ neurites for Elp3cKO + siSCR + DMSO, $18.0 \pm 2.243, n=28$ neurites for Elp3cKO + siSSH + DMSO, $15.22 \pm 1.93, n=25$ neurites for Elp3cKO + siSSH + ML7; tertiary: $5.16 \pm 0.59, n=6$ neurites for Elp3WT + siSCR + DMSO, $4.0 \pm 0.81, n=4$ neurites for Elp3cKO + siSCR + DMSO, $8.0 \pm 2.27, n=4$ neurites for Elp3cKO + siSSH + DMSO, $6.0 \pm 0.40, n=4$ neurites for Elp3cKO + siSSH + ML7; two-way ANOVA, ${ }^{*} P<0.05$; $\left.{ }^{* *} P<0.001\right)$. See also Supplementary information, Figure S3.

trols (Figure 4C). The $\mathrm{G} / \mathrm{F}$ actin ratio depends on a tight balance between regulators of actin polymerization and F-actin severing. Although the main competitive regulators of actin polymerization remain unchanged (Supplementary information, Figure S4J), we observed a significant decrease of cofilin phosphorylation in Elp$3 \mathrm{cKO}$ MGE extracts in vivo (Figure 4D). Cofilin is the predominant actin-severing enzyme, which is inhibited by phosphorylation. Interestingly, cofilin and Elongator subunits interact in both MGE and N2A cells (Figure 4E, $4 \mathrm{~F}$ and Supplementary information, Figure S3D), a process that may contribute to the molecular regulation of cofilin phosphorylation (Figure 4D and Supplementary information, Figure S3E). To test whether decreased phopshorylation of cofilin accounts for the migration defects of Elp3cKO interneurons, we blocked cofilin dephosphorylation by performing electroporation of MGE with esiRNAs (Figure 4G) against the cofilin phosphatase Slingshot homolog 2 (SSH2; Supplementary information, Figure S4K), the predominant Slingshot homolog family member expressed in migrating cortical interneurons (Supplementary information, Figure S4L). Importantly, expression of the three SSH members remained unchanged upon loss of Elp3 expression (Supplementary information, Figures S4M-S4O). Live imaging showed that SSH2 knockdown rescued the velocity of Elp3cKO interneurons (Figure 4H), their nucleokinesis parameters (Figure 4I-4K), as well as the formation of new dynamic branches from the growth cone (Figure $4 \mathrm{~L}$ and $4 \mathrm{M}$ ). The extension of the leading process was only rescued by the concomitant depletion of SSH2 together with inhibition of MLCK (Figure 4N; see also Supplementary information, Figure $3 \mathrm{~J}$ ). However, such treatment had no positive synergistic effect on other migratory events as well as the length of the secondary neurites in Elp3cKO interneurons (Figure 4I-4N and Supplementary information, Movies S5-S8). Except for the trailing process, the somal distribution of actomyosin in Elp3cKO interneurons was greatly improved by esiSSH2 with or without ML-7 treatments, as suggested by the decreased duration of non-polarized events and the increased number of events behind the nuclear rear in Elp3cKO interneurons (Figure $5 \mathrm{~A}$ and 5B). Accordingly, immunolabelings revealed that targeting $\mathrm{SHH} 2$ promoted the perinuclear relocalization of p-cofilin (Figure 5C-5E) and reduced the G/F-actin ratio in all cellular compartments of migrating Elp3cKO interneurons (Figure 5F and 5G).

Thus, our results show that the genetic deletion of 
A

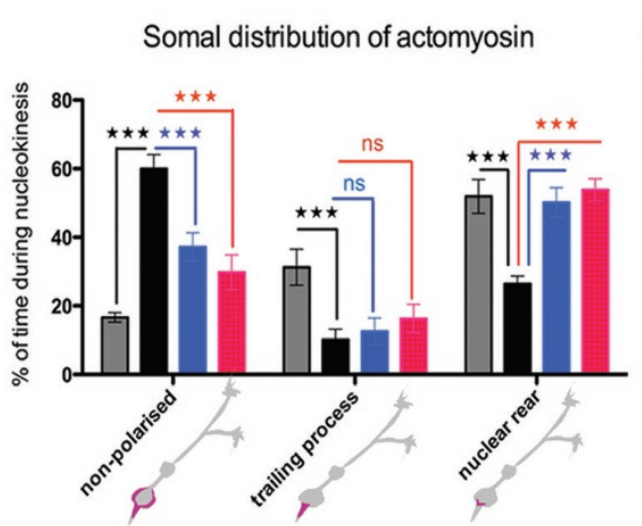

C

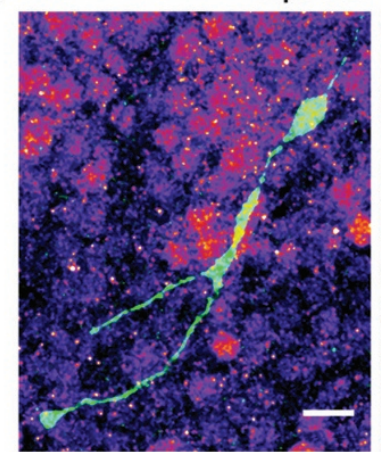

Elp3 WT + siSCR

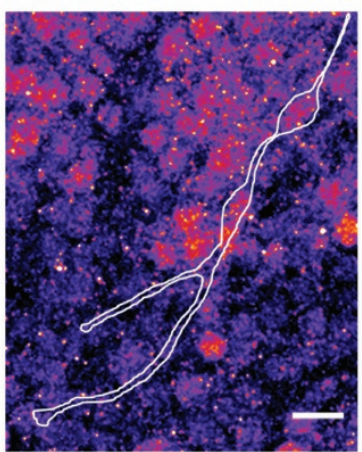

E

$\mathrm{Elp} 3 \mathrm{cKO}+\mathrm{siSHH}$
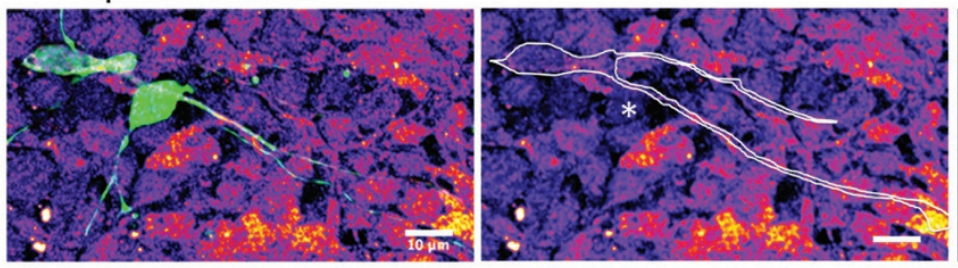

D

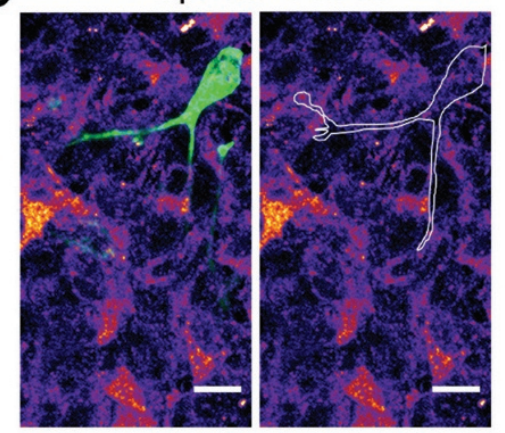

B LifeAct

Elp3 $\mathrm{CKO}+$ siSCR+DMSO

- Elp3cKO + siSCR + DMSO

Elp3cKO + siSSH + DMSO

Elp3cKO + siSSH + ML-7

p-cofilin / GFP / RFP
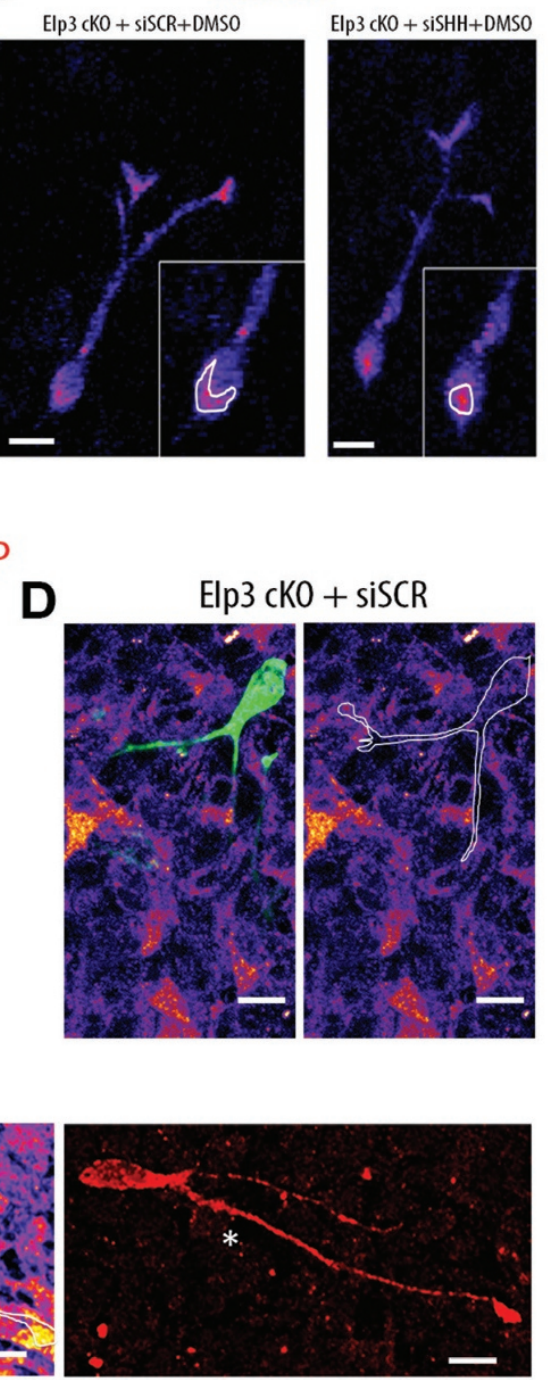

$\mathbf{F}$

$$
\text { F-actin/ G-actin }
$$

Elp3 L/L + Cre +sisCR Elp3 L/L + Cre +siSHH

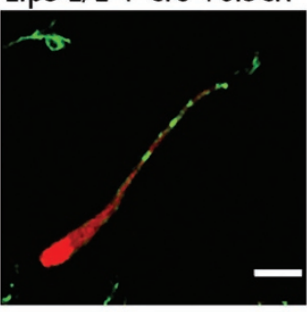

G

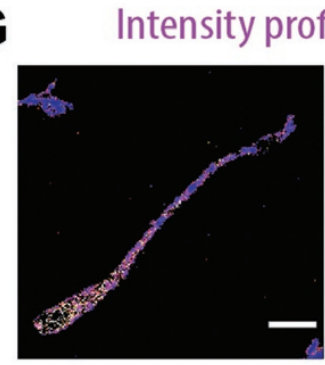

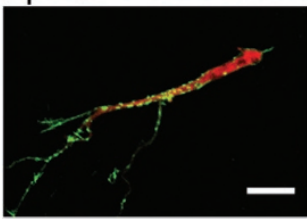

file of $\mathrm{G} / \mathrm{F}$-actin
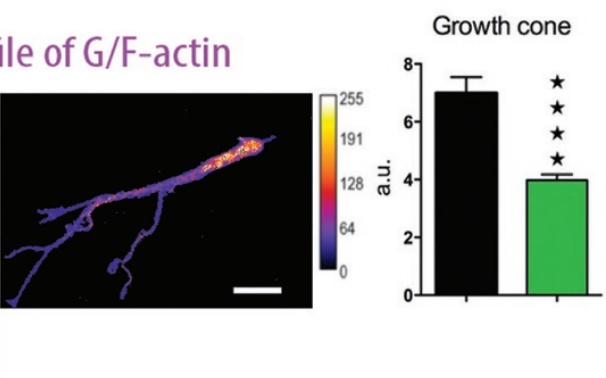
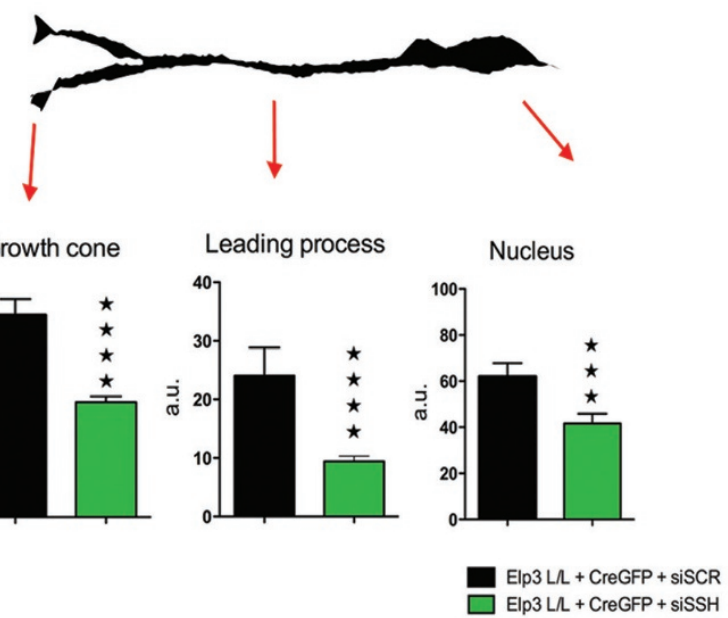
Figure 5 Targeting $\mathrm{SHH} 2$ rescues the actin-severing defects and improves actomyosin parameters in Elp3cKO interneurons. (A, B) Somal distribution of actomyosin is sensitive to SSH2 knockdown ( ${ }^{* \star * *} P<0.0001$ (interaction) two way ANOVA and Bonferroni's post hoc test $n=9-11$ cells in three independent experiments) in migrating interneurons, genotypes and treatment as indicated on histograms. (C-E) Impaired p-cofilin expression is partially rescued by SSH2 knockdown in Elp3cKO interneurons. Single confocal plan imaging showing Dlx5, 6-GFP positive interneurons expressing or not Elp3 (GFP, green) focally electroporated with RFP (red) and esiSCR (C, D) or esiSSH2 (E). The star in panel E shows lack of p-cofilin rescue in GFP interneurons non-electroporated with esiSSH2. (F-G) Targeting SHH2 reduced the G/F-actin ratio in Elp3 acutely depleted interneurons. Immunolabelings for F-actin (phaloidine, green) and G-actin (anti-DNAse I, red) in migrating Elp3L/L cortical interneurons focally electroporated with Cre and esiSCR or esiSSH2 (F). Intensity profiles of G/F-actin (fire) measured at different parts of the cell (G). Scale bars represent $10 \mu \mathrm{m}$ (B-G). See also Supplementary information, Figure S4.

Elp3 results in a coincident decrease of p-cofilin and accumulation of non-polarized actin microfilaments (as detected by LifeAct) in cortical interneuron soma. This molecular phenotype, together with the overall increase of MLC phosphorylation, results in less efficient actomyosin forces to control nulceokinesis and growth cone splitting during tangential migration of cortical interneurons (Figure 6). According to its ability to bind to cofilin, an interaction that may take place on or close to actin microfilaments (Supplementary information, Figure $\mathrm{S} 4 \mathrm{P}$ ), we suggest that Elongator may control actomyosin dynamics by promoting phosphorylation of cofilin in migrating cortical interneurons.

\section{Discussion}

The complex organization of the cerebral cortex reflects the exquisite choreography of neuron movements that take place during its development. Cortical interneurons migrate from the subpallium over long distances to integrate into the developing cortical wall. They undergo dynamic morphological remodeling that requires the fine regulation of their cytoskeleton. The present work highlights a novel cell-autonomous but non-catalytic role of Elongator for proper migration of cortical interneurons. At the molecular level, the conditional loss of Elongator in interneurons impairs cofilin activity, leading to increased actin severing, which disrupts actomyosin contractility in the soma and in growth cone. Converging experimental and clinical evidence suggest that altered interneuron migration contributes to neurological disorders such as schizophrenia and autism (reviewed in [42]). Defining how interneurons migrate and integrate into specific cortical networks is, therefore, essential for understanding the biological basis of these disorders.

\section{Elongator controls neuron migration in the forebrain}

During embryogenesis, the migration of neurons is a critical process for proper neuronal positioning and for establishing cortical wiring. Navigation of cortical interneurons into the forebrain parenchyma involves the interplay between cell extrinsic molecular cues and intrinsic mechanisms that coordinate the morphological remodeling with cell movement. Most signals converge on the dynamic control of cytoskeletal elements and their regulators to support migration [13, 43-47]. The reduction of Elongator activity impairs the migration of excitatory projection neurons in the developing cerebral cortex, partly by preventing proper acetylation of $\alpha$-tubulin in MTs [15]. Surprisingly, such a defect was not observed in Elp3cKO interneurons, suggesting the presence of complementary molecular mechanisms for the loss of Elongator activity in cortical interneurons [48]. Although Elp1 controls the subcellular distribution of the actin organizer FlnA in cultured MEFs and HeLa cells [40], the conditional loss of Elp3 expression in cortical interneurons does not modify the expression level or the subcellular distribution of FlnA. However, the loss of Elp3 correlates with a disorganization of the actin network. Indeed, our results show that Elongator underlies the migration of cortical interneurons through modulation of the F-actin-severing activity of cofilin. Cofilin regulates actin dynamics, and its phosphorylation, which inhibits its activity, is critical for the proper formation of actomyosin filaments that provide forces during motility. The accumulation of pMLC may occur in Elp3cKO interneurons to increase the overall activity of myosin II as a molecular mechanism to compensate for the poor spatial distribution and efficiency of actomyosin filaments. This mechanism may also be reinforced by the enhanced recruitment of MLC to F-actin upon loss of Elp3 expression (Supplementary information, Figure S4P).

Although the loss of Elongator results in dephosphorylation of cofilin, it remains unclear how this complex controls cofilin activity. Although Elongator subunits bind to cofilin in vitro and in vivo, regulation of its activity likely depends on the recruitment of its kinase (e.g., LIMK) and/or some phosphatases (e.g., Slingshot homologs, SHH1-3). Given the lack of interaction of Elongator subunit with LIMK (data not shown) and that no modifications of LIMK activity (Supplementary information, Figures S4I) were detected upon loss of Elp3 in MGE 


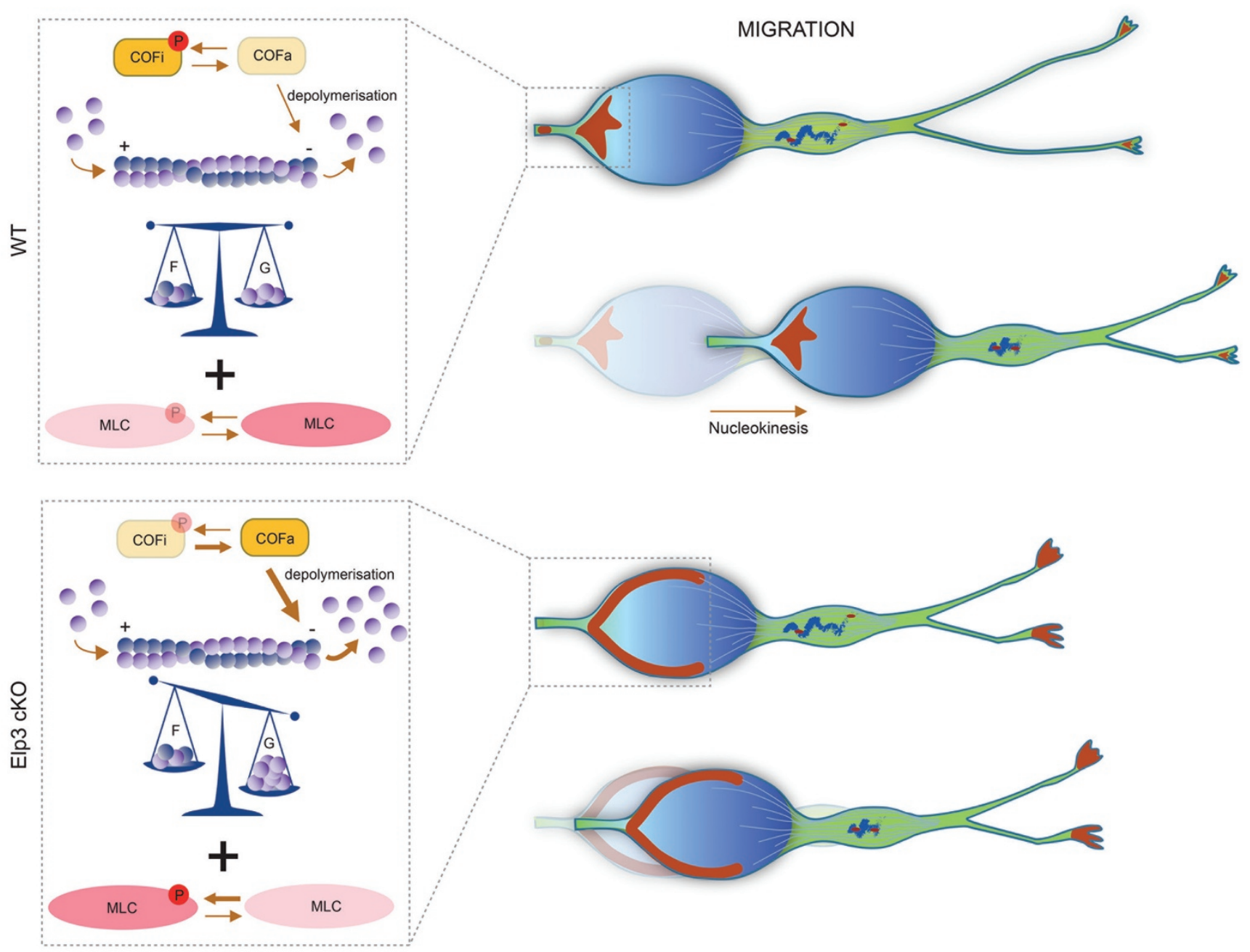

Figure 6 Summary scheme of the molecular pathways downstream of Elongator that contribute to the migration of cortical interneurons in mice. When Elp3 is expressed, cofilin has a basal level of activity (equilibrium between COFi and COFa) that allows proper formation of F-actin, which together with the activation of the myosin II motors (via the MLCK-dependent phosphorylation of MLC II) ensures proper nucleokinesis and branching of interneurons. Loss of Elp3 expression results in the unbalanced activity of cofilin via reduction of its phosphorylation, which further increases the severing of F-actin. This in turn impairs the formation of the actin cytoskeleton and the spatial regulation of actomyosin forces (red signal in the soma and growth cone of interneurons). The increased phosphorylation of MLC (deep pink) may result from the disruption of the actin cytoskeleton and the upregulation of its main kinase MLCK (see text).

extracts (data not shown), we propose that Elongator modulates the cofilin activity by preventing its interaction with SHHs. Interestingly, this function of Elongator does not require the Elp3 KAT activity as suggested by rescue experiments performed with expression of the Elp3mKAT plasmid in Elp3cKO interneurons (Figure $2 \mathrm{R}-2 \mathrm{U}$ ). It is noteworthy that the quantitative difference of rescue of the migratory parameters upon expression of Elp3 or Elp3KAT may result from their different efficiency of integration into the Elongator complex.

Moreover, Elp3 and Elp1 are found to be enriched with MLC in the F-actin fraction of MGE extracts (Supplementary information, Figure S4P), suggesting that, in vivo, Elongator may sit on actin microfilaments to locally control their polymerization and spatially regulate the actomyosin dynamics required for tangential migration. Accordingly, we propose that Elongator acts as a scaffold nearby actin filaments (Supplementary information, Figure S4P) for the recruitment of cofilin and its activity regulators.

In addition to F-actin defects, we observed the upregulation of MLCK together with accumulation of pMLC in 
MGE extracts from E14 Elp3cKO embryos, as compared with their WT controls. The overexpression of MLCK in Elp3cKO interneurons may result from the disruption of its actin networks. The inability to rescue the migration defects by pharmacologically blocking MLCK further supports the disruption of cofilin activity and thus the G/ F-actin balance as the major driver of the migration phenotype. However, we cannot rule out the possibility that loss of Elp3 expression in cortical interneurons may also impair the activity of other actin regulators such as the Rho-regulated actin nucleators mammalian diaphanous homolog 1 (mDia1) and 3 (mDia3), whose contribution to migration was recently demonstrated for postnatal neuroblasts invading the rostral migratory stream [49].

\section{Multiple roles for Elongator during the establishment of the cerebral cortex}

The Elongator complex is widely distributed in the forebrain, from neuronal stem cells to differentiating neurons in the cortical plate of the maturating cerebral cortex. Loss or reduction of its activity has been associated with migration defects of cortical neurons in vivo [15]. The migration of projection neurons and interneurons in the developing cerebral cortex relies on the dynamic remodeling of their cytoskeleton. Disrupting the regulation of either actin or MTs has been shown to impair neuronal migration in the cortex [11, 50-52].

While Elongator promotes the migration of excitatory projection neurons by ensuring proper acetylation of MTs [15], we now show that this modification is not altered upon genetic deletion of Elp 3 in cortical interneurons. This suggests that the migration of cortical projection neurons and interneurons may be more dependent on the fine regulation of the MT or the actin cytoskeleton, respectively. Indeed, tight control of the actin dynamics and the distribution of actomyosin forces are critical for proper navigation of interneurons in the forebrain parenchyma $[10,11,53]$. Although the actin cytoskeleton is not prominently affected upon Elp3 depletion in projection neurons that are settling in the cortical plate [15], fine regulation of $\mathrm{F} / \mathrm{G}$-actin ratio is still required for their migration $[54,55]$. One possible reason why actin-related defects were not observed in projection neurons may be due to their distinct modes of migration as compared with interneurons. Indeed, when analyzed at E14.5, projection neurons migrate radially predominantly via locomotion on radial glia cell (RGC) fibers. This locomotion of projection neurons along RGC fibers requires a more stable morphology that mostly involves MT regulation by its associated proteins $[15,56,57]$. In contrast, interneurons migrate tangentially, mostly relying on actomyosin dynamics to support nucleokinesis, dynamic branch- ing of their leading process $[10,11,53,58]$ and their direction of migration [59]. We should, thus, reinvestigate the impact of Elp3 deletion on the actin cytoskeleton and actomyosin dynamics of migrating projection neurons by specifically focusing on the transition between multipolar and bipolar stages that more critically depend on actin regulation [60].

We recently demonstrated that loss of Elp3 impairs cortical neurogenesis by modifying the fate choice of apical progenitors (APs) in the developing cortex. Elp3 is a tRAMEs [30] and loss of its activity impaired codon translation speed that triggers ER stress and activation of the PERK branch of the UPR in APs [29]. Although ER stress can interfere with cell migration [37], we were unable to detect the molecular readout of its activation in Elp3cKO-migrating neurons (Supplementary information, Figure S2C and S2D). This may be due to differing rates of protein synthesis between postmitotic neurons and their progenitors.

\section{Materials and Methods}

\section{Animals}

The Elp3 gene was specifically disrupted in cortical interneurons by homologous recombination with the Cre-LoxP gene targeting system [61]. Elp3 conditional knockout mice were generated by backcrossing Elp3loxp/loxp [29, 31] into the MF1 background of Dlx 5,6:Cre-GFP (Dr K Campbell, Cincinnati, USA) for selected experiments and housed under standard conditions at the GIGA-Mouse facility and Transgenics, University of Liège. All animals were treated according to the guidelines of the Belgian Ministry of Agriculture in agreement with European community Laboratory Animal Care and Use Regulations (86/609/CEE, Journal Officiel des Communautées Européennes, L358, 18 December 1986). PCR-based genotyping was performed with primers Elp3_1 (5'-GCAAACTGACCTAAAGCTACC-3') and Elp3 2 (5'-TTCCTTCGGTTTCTGTGACC-3') and for genotyping Cre, CreFwd (5'-ATCCGAAAAGAAAACGTTGA-3') and CreRev (5'-ATCCAGGTTACG GATATAGT-3').

\section{Electroporation}

Electroporations of MGE were performed as previously described with minor modifications [62]. Briefly, endofree plasmid DNA solutions $(2 \mu \mathrm{g} / \mu \mathrm{l}$, Qiagen), mixed with $0.05 \%$ Fast Green (Sigma, St Louis, USA), were injected into the MGE of E13.5 brains slices or directly (ex vivo) into the MGE from E13.5 embryos. Electroporations were conducted with platinum electrodes (CUY650P3 and CUY701P7E, Sonidel) using ECM-830 BTX square wave electroporator (VWR International, Leuven, Belgium) 5 pulses of $100 \mathrm{~V}$ of $10 \mathrm{~ms}$ duration at $1 \mathrm{~s}$ intervals were used for focal electroporation on brain slices and 5 pulses of $50 \mathrm{~V}$ of $50 \mathrm{~ms}$ duration at $1 \mathrm{~s}$ were used for the ex vivo electroporation.

\section{Time lapse imaging}

Time lapse images of $\mathrm{GPF}^{+}$interneurons of slices and explants or RFP-expressing migrating interneurons of focally electroporated slices were acquired every $5 \mathrm{~min}$ during $5 \mathrm{~h}$. Images of brain 
slices were taken in dorso-lateral regions of the cortex with 18 " $\mathrm{z}$ " plans of $1.5 \mu \mathrm{m}$. Images of ex vivo electroporation of the MGE with LifeAct-RFP plasmid with or without esiRNA SHH RNA were acquired every $20 \mathrm{~s}$ for 3-4 h with resonant scanning and xyzt acquisition modes. Nuclear translocations were considered as significant movement when over $5 \mu \mathrm{m}$. The amplitude of translocation corresponds to the average significant nuclear movement per cell (independent of the existence of a swelling). The frequency of nuclear translocation corresponds to the number of significant nuclear displacement divided by the time of recording. LifeAct electroporated interneurons were analyzed with the fire-mode in ImageJ to visualize localization and intensity changes.

\section{Western blotting analyses}

Microdissected GEs were incubated in the lysis buffer $(50 \mathrm{mM}$ Tris- $\mathrm{HCl}, \mathrm{pH} 7.4,450 \mathrm{mM} \mathrm{NaCl}, 1 \%$ triton, $10 \mathrm{mM} \mathrm{NaF}, 1 \mathrm{mM}$ $\mathrm{Na}_{3} \mathrm{VO}_{4}$ and proteases inhibitors, (Roche)) and sonicated. Cellular extracts were cleared by centrifugation $(10000 \times g)$ for $10 \mathrm{~min}$ at $4{ }^{\circ} \mathrm{C}$ and 10-30 $\mu \mathrm{g}$ (2-4 $\mu \mathrm{g}$ for tubulin/acetyl tubulin WB) of proteins were separated by SDS-PAGE and transferred to $0.45 \mu \mathrm{m}$ nitrocellulose membranes (GE Healthcare life sciences, Germany). Membranes were blocked for $30 \mathrm{~min}$ in $5 \%$ non-fat milk PBS$0.1 \%$ Tween 20 and incubated overnight at $4{ }^{\circ} \mathrm{C}$ with specific primary antibodies followed by incubation with HRP-conjugated secondary antibodies for $2 \mathrm{~h}$ at room temperature (HRP-conjugated anti-mouse IgG, anti-mouse, anti-rabbit, anti-goat, 1:10 000, GE Healthcare, Waukesha, USA). Membranes were developed using Hyperfilm ECL (GE Healthcare, Waukesha, USA) with the ECL chemiluminescent reagent (Thermo Scientific, Rockford, USA). The following antibodies were used for western blot: rabbit anti-Elp3 (1:500, Cell Signaling, Danvers, USA), mouse anti-Elp3 (1:500, Abcam, USA), rabbit anti-RhoA (1:500, NewEast Biosciences, Malvern, USA), mouse anti-RhoA (1:200, Santa Cruz, USA), rabbit anti-ser3phosphocofilin (1:500, Cell Signaling), rabbit anti-cofilin (1:500, Cell Signaling, Danvers, USA), mouse anti-phosphomyosin light chain II (1:500 Cell Signaling), rabbit anti-myosin light chain II (1:500, Cell Signaling), mouse anti myosin light chain kinase (MLCK; 1:1 000, Sigma, St Louis, USA), mouse anti- $\alpha$-tubulin (1:30 000, Sigma), mouse anti-acetylated $\alpha$-tubulin (1:30 000, Sigma), rabbit anti-phospho PAK1/PAK2 (1:500, Cell Signaling), rabbit anti-PAK (1:250, Santa Cruz), rabbit anti-filamin A (1:10 000, Abcam), rabbit anti-phospholimkinase 1/2 (1:500, Novus Biologicals, Colorado, USA), rabbit-limkinase $1 / 2$ (1:500, Cell Signaling) and mouse anti- $\beta$ actin peroxidase $(1: 10$ 000, Sigma).

\section{Immunoprecipitations}

Microdissected GEs were incubated in lysis buffer $(50 \mathrm{mM}$ Tris-HCl, pH 7.5, 1 mM EDTA, 1\% NP40, $150 \mathrm{mM} \mathrm{NaCl}$, proteases inhibitors (Roche)). MGE extracts were cleared by centrifugation $\left(13000 \times g, 10 \mathrm{~min}, 4^{\circ} \mathrm{C}\right)$. Coimmunoprecipitations were carried out with $500-800 \mu \mathrm{g}$ proteins that were pre-cleared with protein A-agarose beads or G plus-agarose beads (Santa Cruz, USA) before incubation with specific antibodies overnight at $4{ }^{\circ} \mathrm{C}$ and then immunoprecipitated by $3-\mathrm{h}$ of incubation with protein A-agarose beads or G plus-agarose beads at $4{ }^{\circ} \mathrm{C}$. Anti-HA or anti-Flag immunoprecipitation was carried out as negative control on microdissected GEs. The following antibodies were used: mouse anti-Flag (1:250, Santa Cruz, USA), rabbit anti-HA (1:250, Santa
Cruz, USA), mouse anti-Elp3 (Abcam, USA), rabbit anti-Elp1 (home-made) and rabbit anti-cofilin. Immunoprecipitates were then subjected to SDS-PAGE for subsequent western blotting analysis.

RhoA-GTP immunoprecipitations were carried out following the manufacturer's instructions (mouse anti-RhoA-GTP, 1:250, NewEast Biosciences, Malvern, USA) and western blotting analysis was performed using mouse anti-RhoA (1:100, Santa Cruz, USA).

\section{F-actin sedimentation assay}

Microdissected GEs (8 GEs per $500 \mu \mathrm{l})$ or $48 \mathrm{~h}$ post-transfection N2A cells were solubilized in sedimentation assay buffer $(50$ mM PIPES, $\mathrm{pH} 6.9,50 \mathrm{mM} \mathrm{NaCl}, 5 \mathrm{mM} \mathrm{MgCl}_{2}, 5 \mathrm{mM}$ EGTA, $5 \%$ glycerol, $0.1 \%$ NP40, $0.1 \%$ Triton X-100, 0.1\% Tween $20,0.1 \%$ $\beta$-mercaptoethanol) incubated for $10 \mathrm{~min}$ at $37^{\circ} \mathrm{C}$ and centrifuged (2 $000 \times g, 5 \mathrm{~min}$ ) to remove nuclei. The supernatants were then subjected to a high-speed centrifugation step $(100000 \times g, 1 \mathrm{~h}, 37$ $\left.{ }^{\circ} \mathrm{C}\right)$ to obtain a clear supernatant (G-actin) and a pellet (F-actin). Pellets were dissolved (water containing $10 \mu \mathrm{M}$ cytochalasin D) in the same volume as the collected supernatant and incubated overnight at $4{ }^{\circ} \mathrm{C}$. Equal volumes of each fraction were subjected to western blotting analysis.

\section{Cell culture}

MEFs were isolated [11] from E13.5 Elp3 ${ }^{\text {lox/lox }}$ embryos [29] and infected with lenti-GFP or lenti-cre viruses, to obtain control or Elp3KO MEFs. MEFs were cultivated in DMEM/F12, 10\% fetal bovine serum (FBS), $2 \mathrm{mM}$ L-glutamine, 1\% penicillin/streptomycin $(\mathrm{P} / \mathrm{S})$. Briefly, to generate lenti-cre vector, the cDNA encoding the cre-recombinase was amplified by PCR from the PCAGGS NeuroD-cre and then inserted into the Nhe1/Kpnl restriction sites of pLenti-CMV-2A-GFP vector (GENTAUR, Poland). Lentiviral stocks were performed at the GIGA-viruses platform according to their own protocols (GIGA-R, ULg, Belgium).

HEK293 cells were expanded in DMEM, 10\% FBS, $20 \mathrm{UI} /$ $\mathrm{ml} \mathrm{P} / \mathrm{S}$ and transfected with Lipo2000 reagents, according to the manufacturer's instructions, with a FLAG-RFP construct (control), a Flag-Elp3-RFP construct or a Flag-Elp3Y529A-RFP-mutant construct. After 2 days of culture, cells were rinsed with PBS, collected, centrifuged and the pellet was snap frozen until western blotting analysis.

N2A cells were expanded in DMEM/F12, 10\% FBS, $20 \mathrm{UI} /$ $\mathrm{ml} \mathrm{P/S}$ and transfected with Lipo2000 reagents, according to the manufacturer's instructions with a esiSCR (D-001810-10-05, GE Dhermacon) or esiElp3 (EMU087711, Sigma) at 70\% confluence. After $6 \mathrm{~h}$ the medium was changed and scratches (three per well) were performed using a p100 tips $24 \mathrm{~h}$ post transfection at $100 \%$ confluence. Pictures were acquired (Evos FL, Life Technologies) at three different time points $(0 \mathrm{~h}, 24 \mathrm{~h}$ and $48 \mathrm{~h})$. Wound healing analysis was performed by measuring the distance of the scratch at each time point divided by the original scratch distance.

Imaging of migrating interneurons was performed on MGE explants cultured onto homochronic layer of E13.5 cortical neurons or organotypic slices from Elp3WT or Elp3cKO embryos as previously described [11]. Briefly, these MGE explants and brain slices were cultured for $24 \mathrm{~h}$ before time lapse recordings in Neurobasal medium, $2 \mathrm{mM}$ L-glutamine, $33 \mathrm{mM}$ D-glucose, $3 \mathrm{mM}$ sodium bicarbonate, $10 \mathrm{mM}$ HEPES buffer, $5 \mathrm{mM}$ Pyruvate, 20 
UI penicillin streptomycin, 1\% N2 and 2\% B27 complement with vitamin A. For some experiments, focal or ex vivo electroporations of the MGEs with esiSCR (D-001810-10-05, GE Dhermacon) or esiSSH2 (EMU046241, Sigma) were performed before starting the culture and then cultured for $24 \mathrm{~h}$ before time lapse recordings.

\section{Cell counting, analyses and statistics}

$\mathrm{GFP}^{+}$interneurons were counted in the cortex at E12.5 and E14.5 in distinct cortical regions of $200 \mu \mathrm{m}$ divided in 10 equal bins at E14.5 and E18. Analyses are performed on maximum intensity Z-projection and migrating cells are tracked using MTrackJ plugin on at least three different experiments with littermates. All graphs are mean \pm SEM. Statistics for dual comparisons were generated using unpaired Student's $t$-tests, and statistics for multiple comparisons were generated using one-way ANOVA or two-way ANOVA followed by Dunnet's, Bonferroni's or Tukey's post hoc test (GraphPad Prism software, version 6, San Diego, USA); ${ }^{*} P<$ $0.05,{ }^{* *} P<0.01,{ }^{* *} P<0.001$, and ${ }^{* * *} P<0.0001$ for all statistics herein.

\section{Acknowledgments}

We thank Dr Kenneth Campbell for providing the Dlx5, 6:CreGFP transgenic mouse line. We thank Drs Creppe and Gladwyn-Ng for critical reading of the manuscript. ST is a $\mathrm{PhD}$ fellow supported by the Belgian Fonds pour la Formation à la Recherche dans l'Industrie et dans l'Agriculture (FRIA). JDG is a postdoctoral researcher from the FRS- FNRS; LN is a Research Associate from FRS-FNRS; AC and BM are Research Directors of the FRSFNRS. JDG has been granted Marie Curie and EMBO LT fellowships. LN is funded by FRS-FNRS, the Fonds Léon Fredericq, the Fondation Médicale Reine Elisabeth, the Fondation Simone et Pierre Clerdent and the Belgian Science Policy (IAP-VII network P7/20). LN and AC are funded by ARC (ARC11/16-01) and partly funded by WELBIO.

\section{Author Contributions}

ST, SH and LN designed the study. ST and SH phenotyped Elp$3 \mathrm{cKO}$ mice, performed time-lapse recordings and biochemistry; ST performed all electroporations. ST, SH, and LN contributed to data analysis and interpretation. AC shared reagents; AC and BM provided critical input on the results. $\mathrm{LN}$ wrote the paper with help from ST, SH, JDG and input from all other co-authors.

\section{Competing Financial Interests}

The authors declare no competing financial interests.

\section{References}

1 Rash BG, Grove EA. Area and layer patterning in the developing cerebral cortex. Curr Opin Neurobiol 2006; 16:25-34.

2 Gupta A, Tsai LH, Wynshaw-Boris A. Life is a journey: a genetic look at neocortical development. Nat Rev Genet 2002; 3:342-355.

3 Anderson SA, Eisenstat DD, Shi L, Rubenstein JL. Interneuron migration from basal forebrain to neocortex: dependence on Dlx genes. Science 1997; 278:474-476.

4 Gelman DM, Martini FJ, Nobrega-Pereira S, Pierani A, Kes- saris N, Marin O. The embryonic preoptic area is a novel source of cortical GABAergic interneurons. J Neurosci 2009; 29:9380-9389.

5 Wonders CP, Anderson SA. The origin and specification of cortical interneurons. Nat Rev Neurosci 2006; 7:687-696.

6 Metin C, Baudoin JP, Rakic S, Parnavelas JG. Cell and molecular mechanisms involved in the migration of cortical interneurons. Eur J Neurosci 2006; 23:894-900.

7 Tanaka DH, Maekawa K, Yanagawa Y, Obata K, Murakami F. Multidirectional and multizonal tangential migration of GABAergic interneurons in the developing cerebral cortex. Development 2006; 133:2167-2176.

8 Marin O, Plump AS, Flames N, Sanchez-Camacho C, Tessier-Lavigne M, Rubenstein JL. Directional guidance of interneuron migration to the cerebral cortex relies on subcortical Slit1/2-independent repulsion and cortical attraction. Development 2003; 130:1889-1901.

9 Marin O, Valiente M, Ge XC, Tsai LH. Guiding neuronal cell migrations. Cold Spring Harb Perspect Biol 2010; 2:a001834.

10 Bellion A, Baudoin JP, Alvarez C, Bornens M, Metin C. Nucleokinesis in tangentially migrating neurons comprises two alternating phases: forward migration of the Golgi/centrosome associated with centrosome splitting and myosin contraction at the rear. J Neurosci 2005; 25:5691-5699.

11 Godin JD, Thomas N, Laguesse S, et al. p27(Kip1) is a microtubule-associated protein that promotes microtubule polymerization during neuron migration. Dev Cell 2012; 23:729-744.

12 Etienne-Manneville S. Microtubules in cell migration. Annu Rev Cell Dev Biol 2013; 29:471-499.

13 Heng JI, Chariot A, Nguyen L. Molecular layers underlying cytoskeletal remodelling during cortical development. Trends Neurosci 2010; 33:38-47.

14 Poirier K, Lebrun N, Broix L, et al. Mutations in TUBG1, DYNC1H1, KIF5C and KIF2A cause malformations of cortical development and microcephaly. Nat Genet 2013; 45:639647.

15 Creppe C, Malinouskaya L, Volvert ML, et al. Elongator controls the migration and differentiation of cortical neurons through acetylation of alpha-tubulin. Cell 2009; 136:551-564.

16 Anderson SL, Coli R, Daly IW, et al. Familial dysautonomia is caused by mutations of the IKAP gene. Am J Hum Genet 2001; 68:753-758.

17 Nguyen L, Humbert S, Saudou F, Chariot A. Elongator an emerging role in neurological disorders. Trends Mol Med 2010; 16:1-6.

18 Simpson CL, Lemmens R, Miskiewicz K, et al. Variants of the elongator protein 3 (ELP3) gene are associated with motor neuron degeneration. Hum Mol Genet 2009; 18:472-481.

19 Slaugenhaupt SA, Blumenfeld A, Gill SP, et al. Tissue-specific expression of a splicing mutation in the IKBKAP gene causes familial dysautonomia. Am J Hum Genet 2001; 68:598-605.

20 Hawkes NA, Otero G, Winkler GS, et al. Purification and characterization of the human elongator complex. J Biol Chem 2002; 277:3047-3052.

21 Kim JH, Lane WS, Reinberg D. Human elongator facilitates RNA polymerase II transcription through chromatin. Proc Natl Acad Sci USA 2002; 99:1241-1246.

22 Winkler GS, Kristjuhan A, Erdjument-Bromage H, Tempst P, 
Svejstrup JQ. Elongator is a histone H3 and H4 acetyltransferase important for normal histone acetylation levels in vivo. Proc Natl Acad Sci USA 2002; 99:3517-3522.

23 Okada Y, Yamagata K, Hong K, Wakayama T, Zhang Y. A role for the elongator complex in zygotic paternal genome demethylation. Nature 2010; 463:554-558.

24 Close P, Hawkes N, Cornez I, et al. Transcription impairment and cell migration defects in elongator-depleted cells: implication for familial dysautonomia. Mol Cell 2006; 22:521-531.

25 Kristjuhan A, Svejstrup JQ. Evidence for distinct mechanisms facilitating transcript elongation through chromatin in vivo. Embo J 2004; 23:4243-4252.

26 Esberg A, Huang B, Johansson MJ, Bystrom AS. Elevated levels of two tRNA species bypass the requirement for elongator complex in transcription and exocytosis. Mol Cell 2006; 24:139-148.

27 Nedialkova DD, Leidel SA. Optimization of codon translation rates via tRNA modifications maintains proteome integrity. Cell 2015; 161:1606-1618.

28 Rahl PB, Chen CZ, Collins RN. Elp1p, the yeast homolog of the FD disease syndrome protein, negatively regulates exocytosis independently of transcriptional elongation. Mol Cell 2005; 17:841-853.

29 Laguesse S, Creppe C, Nedialkova DD, et al. A dynamic unfolded protein response contributes to the control of cortical neurogenesis. Dev Cell 2015; 35:553-567.

30 Karlsborn T, Tukenmez H, Mahmud AK, Xu F, Xu H, Bystrom AS. Elongator, a conserved complex required for wobble uridine modifications in eukaryotes. RNA Biol 2014; 11:1519-1528.

31 Ladang A, Rapino F, Heukamp LC, et al. Elp3 drives Wnt-dependent tumor initiation and regeneration in the intestine. $J$ Exp Med 2015; 212:2057-2075.

32 Miskiewicz K, Jose LE, Bento-Abreu A et al. ELP3 controls active zone morphology by acetylating the ELKS family member Bruchpilot. Neuron 2011; 72:776-788.

33 George L, Chaverra M, Wolfe L, et al. Familial dysautonomia model reveals Ikbkap deletion causes apoptosis of Pax3+ progenitors and peripheral neurons. Proc Natl Acad Sci USA 2013; 110:18698-18703.

34 Jackson MZ, Gruner KA, Qin C, Tourtellotte WG. A neuron autonomous role for the familial dysautonomia gene ELP1 in sympathetic and sensory target tissue innervation. Development 2014; 141:2452-2461.

35 Stenman J, Toresson H, Campbell K. Identification of two distinct progenitor populations in the lateral ganglionic eminence: implications for striatal and olfactory bulb neurogenesis. J Neurosci 2003; 23:167-174.

36 Wittschieben BO, Fellows J, Du W, Stillman DJ, Svejstrup JQ. Overlapping roles for the histone acetyltransferase activities of SAGA and elongator in vivo. Embo J 2000; 19:30603068.

37 Saez PJ, Villalobos-Labra R, Westermeier F, Sobrevia L, Farias-Jofre M. Modulation of endothelial cell migration by ER stress and insulin resistance: a role during maternal obesity? Front Pharmacol 2014; 5:189.

38 Chrzanowska-Wodnicka M, Burridge K. Rho-stimulated contractility drives the formation of stress fibers and focal adhesions. J Cell Biol 1996; 133:1403-1415.
39 Burkel BM, von Dassow G, Bement WM. Versatile fluorescent probes for actin filaments based on the actin-binding domain of utrophin. Cell Motil Cytoskeleton 2007; 64:822-832.

40 Johansen LD, Naumanen T, Knudsen A, et al. IKAP localizes to membrane ruffles with filamin A and regulates actin cytoskeleton organization and cell migration. J Cell Sci 2008; 121:854-864.

41 Nakamura F, Osborn TM, Hartemink CA, Hartwig JH, Stossel TP. Structural basis of filamin A functions. J Cell Biol 2007; 179:1011-1025.

42 Marin O. Interneuron dysfunction in psychiatric disorders. Nat Rev Neurosci 2012; 13:107-120.

43 Chenn A, Walsh CA. Regulation of cerebral cortical size by control of cell cycle exit in neural precursors. Science 2002; 297:365-369.

44 Rakic P, Knyihar-Csillik E, Csillik B. Polarity of microtubule assemblies during neuronal cell migration. Proc Natl Acad Sci USA 1996; 93:9218-9222.

45 Rivas RJ, Hatten ME. Motility and cytoskeletal organization of migrating cerebellar granule neurons. J Neurosci 1995; 15:981-989.

46 Schaar BT, McConnell SK. Cytoskeletal coordination during neuronal migration. Proc Natl Acad Sci USA 2005; 102:13652-13657.

47 Xie Z, Samuels BA, Tsai LH. Cyclin-dependent kinase 5 permits efficient cytoskeletal remodeling--a hypothesis on neuronal migration. Cereb Cortex 2006; 16 Suppl 1:i64- i68.

48 Akella JS, Wloga D, Kim J, et al. MEC-17 is an alpha-tubulin acetyltransferase. Nature 2010; 467:218-222.

49 Shinohara R, Thumkeo D, Kamijo H, et al. A role for mDia, a Rho-regulated actin nucleator, in tangential migration of interneuron precursors. Nat Neurosci 2012; 15:373-380.

50 Jaglin XH, Chelly J. Tubulin-related cortical dysgeneses: microtubule dysfunction underlying neuronal migration defects. Trends Genet 2009; 25:555-566.

51 Kappeler C, Saillour Y, Baudoin JP, et al. Branching and nucleokinesis defects in migrating interneurons derived from doublecortin knockout mice. Hum Mol Genet 2006; 15:13871400.

52 McManus MF, Nasrallah IM, Pancoast MM, Wynshaw-Boris A, Golden JA. Lis1 is necessary for normal non-radial migration of inhibitory interneurons. Am J Pathol 2004; 165:775784.

53 Avila A, Vidal PM, Dear TN, Harvey RJ, Rigo JM, Nguyen L. Glycine receptor alpha2 subunit activation promotes cortical interneuron migration. Cell Rep 2013; 4:738-750.

54 Bellenchi GC, Gurniak CB, Perlas E, Middei S, Ammassari-Teule $\mathrm{M}$, Witke $\mathrm{W}$. N-cofilin is associated with neuronal migration disorders and cell cycle control in the cerebral cortex. Genes Dev 2007; 21:2347-2357.

55 Wang JT, Song LZ, Li LL, et al. Src controls neuronal migration by regulating the activity of FAK and cofilin. Neuroscience 2015 ; 292:90-100.

56 Gleeson JG, Lin PT, Flanagan LA, Walsh CA. Doublecortin is a microtubule-associated protein and is expressed widely by migrating neurons. Neuron 1999; 23:257-271.

57 Volvert ML, Prevot PP, Close P, et al. MicroRNA targeting of CoREST controls polarization of migrating cortical neurons. Cell Rep 2014; 7:1168-1183. 
58 Martini FJ, Valdeolmillos M. Actomyosin contraction at the cell rear drives nuclear translocation in migrating cortical interneurons. J Neurosci 2010; 30:8660-8670.

59 Martini FJ, Valiente M, Lopez Bendito G, et al. Biased selection of leading process branches mediates chemotaxis during tangential neuronal migration. Development 2009; 136:41-50.

60 Nagano T, Morikubo S, Sato M. Filamin A and FILIP (Filamin A-Interacting Protein) regulate cell polarity and motility in neocortical subventricular and intermediate zones during radial migration. J Neurosci 2004; 24:9648-9657.

61 Kuhn R, Schwenk F, Aguet M, Rajewsky K. Inducible gene targeting in mice. Science 1995; 269:1427-1429.

62 Tielens S, Godin JD, Nguyen L. Real-time recordings of migrating cortical neurons from GFP and Cre recombinase expressing mice. Curr Protoc Neurosci 2016; 74:3.29.1-23.

(Supplementary information is linked to the online version of the paper on the Cell Research website.) 